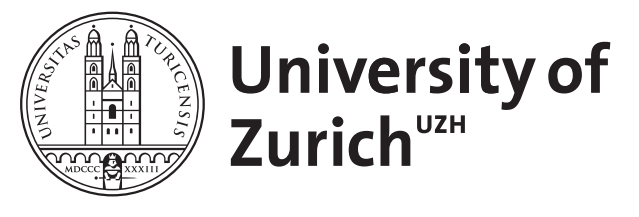

\title{
Flow-based SPPS for protein synthesis: A perspective
}

\author{
Gates, Zachary P ; Hartrampf, Nina
}

\begin{abstract}
Flow-based approaches to solid phase peptide synthesis (SPPS) have been pursued since the method's early days, with anticipated gains in speed, reaction monitoring, and ease of automation. Here, we discuss how these advantages are being realized by synthesis at elevated temperature, facilitated by a 'preheat/activation' loop. This important modification both accelerates peptide synthesis - providing a wealth of new data from in-line monitoring - and in conjunction with an optimized protocol, extends the length of peptides routinely accessible by stepwise synthesis. Streamlined synthesis of longer peptides will address a major challenge in chemical protein synthesis: preparation of peptide segments for use in chemical ligation. The context of recent results in flow-based SPPS is discussed, highlighting remaining challenges and future opportunities.
\end{abstract}

DOI: https://doi.org/10.1002/pep2.24198

Posted at the Zurich Open Repository and Archive, University of Zurich ZORA URL: https://doi.org/10.5167/uzh-193197

Journal Article

Accepted Version

Originally published at:

Gates, Zachary P; Hartrampf, Nina (2020). Flow-based SPPS for protein synthesis: A perspective. Peptide Science, 112(6):e24198.

DOI: https://doi.org/10.1002/pep2.24198 


\title{
Flow-based SPPS for protein synthesis: A perspective
}

\author{
Zachary P. Gates ${ }^{1 *}$, Nina Hartrampf $f^{2 *}$ \\ ${ }^{1}$ p53 Lab, Agency for Science, Technology and Research (A*STAR), 8A Biomedical Grove, \#06-04/05 \\ Neuros/Immunos, Singapore, 138648 \\ ${ }^{2}$ University of Zurich, Department of Chemistry, Wintherthurerstrasse 190, 8057 Zurich, Switzerland \\ * Corresponding authors \\ Email: Zachary_Gates@p53lab.a-star.edu.sg and nina.hartrampf@chem.uzh.ch
}

\begin{abstract}
:
Flow-based approaches to solid phase peptide synthesis (SPPS) have been pursued since the method's early days, with anticipated gains in speed, reaction monitoring, and ease of automation. Here, we discuss how these advantages are being realized by synthesis at elevated temperature, facilitated by a 'preheat/activation' loop. This important modification both accelerates peptide synthesis_-providing a wealth of new data from in-line monitoring — and in conjunction with an optimized protocol, extends the length of peptides routinely accessible by stepwise synthesis. Streamlined synthesis of longer peptides will address a major challenge in chemical protein synthesis: preparation of peptide segments for use in chemical ligation. The context of recent results in flow-based SPPS is discussed, bighlighting remaining challenges and future opportunities.
\end{abstract}

\section{Introduction}

Chemical synthesis is an established means of preparing protein-like molecules comprising noncanonical amino acids and chain topologies. ${ }^{[1]}$ Modern chemical protein synthesis generally employs 'chemical ligation', $[2,3]$ the assembly of protein chains by chemoselective reaction of shorter, unprotected peptide segments containing unique, mutually reactive functional groups. As reviewed elsewhere, the chemical ligation approach leverages both the utility of stepwise solid phase peptide synthesis (SPPS) for obtaining the requisite peptide segments (typically $<50$ amino acid residues), and considerable know-how regarding their characterization and handling ${ }^{[4,5]}$. It has been argued that since the advent of chemical ligation, preparation of unprotected peptide 'building blocks' by means of stepwise SPPS has become the principal bottleneck to chemical protein synthesis in practice. ${ }^{[4,6]}$

In the context of modern protein synthesis, SPPS receives little attention compared to refinements in ligation techniques. ${ }^{[7]}$ Perhaps the focus on ligation chemistries can be attributed to their outsized impact on protein synthesis, and the desire of researchers to stay 'at the forefront'. Figure 1A illustrates the impact of chemical ligation, which was to extend the practical size limit of homogeneous synthetic peptides into the realm of protein domains (reproduced from [4]). In contrast, SPPS dramatically reduced the labor associated with peptide synthesis, but did not significantly extend the length of peptides that were routinely accessible in homogeneous form ${ }^{[5]}$. Considerable effort has gone into optimizing SPPS as currently practiced, and given the mature state of the method, it may be presumed that further advances are unlikely. 
A) Developments in peptide synthesis



B) Developments in stepwise SPPS

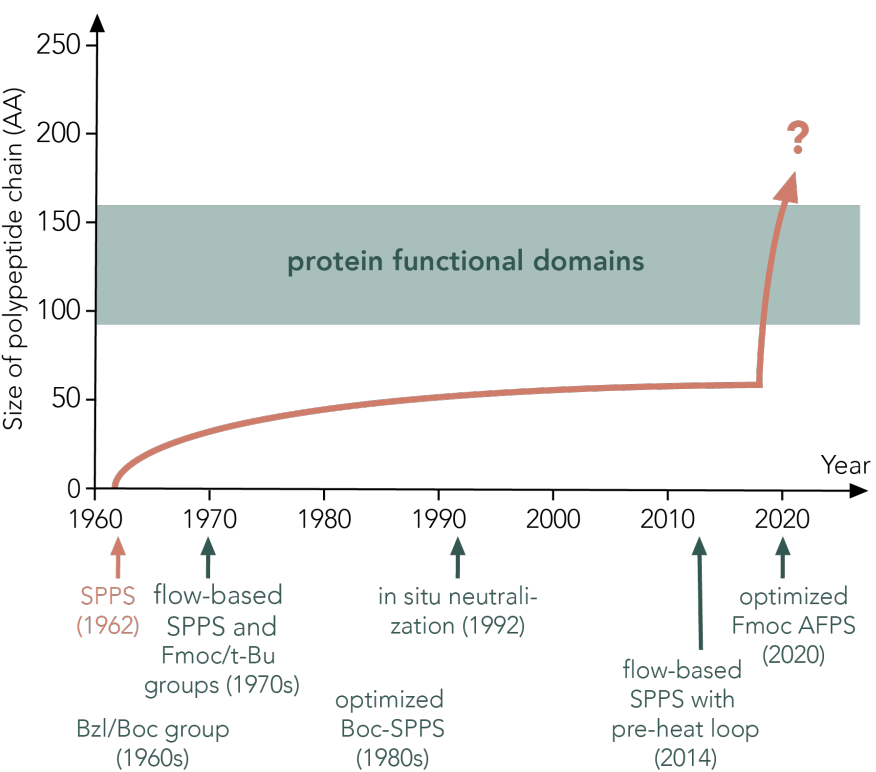

Figure 1 Development of peptide synthesis in the past century. (A) Correlation between the length of synthetic peptides routinely accessible (in amino acids, AA), and important milestones in peptide synthesis. From $P$. E. Dawson, S. B. H. Kent, Annu. Rev. Biochem. 2000, 69, 923-960 [4]. Modified with permission from the corresponding author, reprinted with permission from Annual Reviews. The date 1906 corresponds to publication of Fischer's "Untersuchungen über Aminosäuren, Polypeptide und Proteine"[8]. For a discussion of the history of peptide chemistry, see [9] (B) Correlation between the length of synthetic peptides routinely accessible by stepwise solid phase synthesis (SPPS), and select historical developments. Other important developments, excluded for clarity, include backbone protection and microwave heating [10]. This is a concept figure, intended to convey the relative impact of new methods on the length of synthetic peptides routinely accessed.

Flow chemistry is a substantively different approach to conventional SPPS that is attracting renewed interest, and is poised to impact chemical protein synthesis. ${ }^{[1,12]}$ Historically, interest in flow-based SPPS focused on the prospects of automation, reduced reagent consumption and suitability for realtime monitoring. More recently, the potential of flow-based synthesis to facilitate synthesis at elevated temperature was realized. ${ }^{[12,13]}$ Use of a heated 'activation loop' and reactor facilitates SPPS at elevated temperatures, while maintaining precise control over the conditions of amino acid activation for peptide bond formation. ${ }^{[12]}$ Recent applications of this approach focused primarily on the acceleration of fluorenylmethoxycarbonyl (Fmoc)-chemistry SPPS. Could flow-based synthesis at elevated temperature also facilitate the synthesis of longer chains (Figure 1B)?

In this review, we highlight recent work that tests the limits of flow-based Fmoc-chemistry SPPS for the stepwise synthesis of single-domain proteins, ${ }^{[14]}$ and the historical background necessary to place this work in context. We examine the origins of flow-based SPPS, especially with respect to their motivation and the challenges faced by early adopters, and identify the use of elevated temperature as a recent advance with considerable potential for impact. Temperature facilitates both sequencedependent 'difficult couplings' and rapid synthesis, which in conjunction with real-time quantitative monitoring will drive further improvements in methodology using large-scale datasets. Early results 
suggest that optimized flow-based SPPS at elevated temperature also extends the size of synthetic peptides routinely accessible by stepwise synthesis, with significant implications for chemical protein synthesis. Future directions for flow-based SPPS are also discussed.

\section{Early developments in SPPS under continuous flow conditions}

\subsection{Advantages in principle}

It was realized early on that SPPS would be facilitated, and its automation simplified, if the solid support were fixated in a reactor - historically referred to as "column", because initially HPLCs and HPLC columns were re-purposed for flow-based SPPS. ${ }^{[17,18]}$ The removal of excess reagents by flow washes is faster and more efficient, which shortens the overall synthesis time. Additionally, flow could minimize reagent usage, by reducing the dilution effect that necessarily occurs when reagents are added batch wise to a swollen resin. ${ }^{[19]}$ Hence, a narrow "coupling components band" would result in a high concentration of reagents in the resin bed, facilitating rapid coupling kinetics. Motivated by some of these considerations, a number of research groups pursued the development of flow-based SPPS. ${ }^{[17,20-}$ 22]

Beyond practical considerations, continuous delivery and removal of reagents in flow offers multiple chemical advantages. For example, continuous flow provides a means to leverage reactive/transient intermediates by their continuous delivery to a reactor (flash chemistry), ${ }^{[23]}$ and to reduce exposure of reactor contents to reactive by-products by their continuous removal. In addition, heat transfer is facilitated. As discussed below, these capabilities have been leveraged for flow-based SPPS only recently.

\subsection{Compatibility with solid supports:}

Early adopters of flow-based SPPS expressed concern regarding its compatibility with existing solid support resins. For example, Bayer anticipated that polystyrene resin was unsuitable for use in flowbased tert-butyloxycarbonyl (Boc)-chemistry SPPS, due to its differential swelling under conditions of coupling vs. Boc removal. ${ }^{[17]}$ This anticipated issue was avoided by the use of a non-swelling, silicabased support. A different solution was devised by Erickson, who employed polystyrene resins in conjunction with modified Boc removal conditions to minimize changes in swelling ${ }^{[21]}$. In Fmocchemistry SPPS, swelling changes are avoided altogether by the use of a single solvent (N,Ndimethylformamide, DMF) throughout-but even here, issues arose that were attributed to the poly(dimethylacrylamide) solid support. Sheppard wrote: "On occasions, a previously stable flowing column would begin to compress and shrink in size, ultimately to a volume less than that of the dry unswollen resin. At the same time there was an associated steep rise in pressure". ${ }^{[2]}$ As a solution, kieselguhr-supported polyacrylamide was developed to facilitate flow-based SPPS under conditions of low pressure. ${ }^{[20]}$

In retrospect, it is difficult to establish with certainty the exact cause of high backpressure observed in some early flow-based SPPS applications. However, it is clear that polystyrene resins are not inherently incompatible with flow-based SPPS, since they have since been used by a number of groups in a variety 
of contexts. ${ }^{[25-27]}$ Undesired backpressure could result from a variety of causes, including the presence of fine solids in reagents or resin samples, and due caution must therefore be taken to remove these contaminants before use in flow systems. At present, polyethylene glycol (PEG)-based ChemMatrix $(\mathrm{CM})$ is a popular solid support for use in flow-based SPPS, ${ }^{[1]}$ but others could presumably be used provided fines are removed. In this context, it could be interesting to re-evaluate the microparticulate, mono-sized resin beads originally developed to reduce diffusion time in flow-based SPPS, ${ }^{[1,28]}$ since the majority of synthesis time in recent approaches is spent on washing. ${ }^{[12]}$

\subsection{Time resolved monitoring of Fmoc-chemistry SPPS}

Flow chemistry also allows for non-destructive in-line analysis and therefore provides valuable information on coupling efficiencies and changes in mass transfer. ${ }^{[2]}$ Noteworthy, Sheppard reports that the prospect of real time analytical control was one of the biggest motivations to enter the field of flow chemistry. ${ }^{[30]}$ In-line deprotection (dibenzovulvene)-analysis enabled continuous measurement of ultraviolet (UV) absorbance in Fmoc-based flow synthesis for reaction analysis. In a typical flow synthesis set up (Figure 2A), synthesis solutions were delivered from one or more pumps and transferred to the reactor before consumption of the coupling solution was monitored by in-line Ultraviolet-visible (UV-Vis) spectroscopy. ${ }^{[21]}$ The synthesizer was kept at room temperature and coupling solutions were circulated through a "recycling loop" until coupling was complete. In continuous flow peptide synthesis, spectroscopic monitoring of the reagent stream gives a timedependent elution profile. During the deprotection step, the integral and peak shape of the dibenzofulvene was used early on to gain information on so-called "difficult couplings". The general broadening and flattening of the peaks, which indicates slower deprotection times potentially due to decreased mass transfer, corresponds directly to internal aggregation. ${ }^{[30]}$ Erickson noticed that "in principle, such monitoring data could serve as the information needed for real-time feedback control of an automated continuous-flow synthesizer". ${ }^{[21]}$ Notably, the general concept of feedback in flow for autonomous process optimization is a concept that has only recently reached its full potential. ${ }^{[31]}$

Pressure monitoring can be used to investigate resin volume changes, often referred to as "Swellographics". A correlation of aggregation and resin volume changes was reported multiple times in the literature. ${ }^{[32,33]}$ To monitor these events in flow, a reactor was developed which adjusted to the volume of the resin by a tightly sealed moving piston. The piston was pressurized by a controlled force and real-time swelling behavior was recorded. ${ }^{[34,35]}$ Rodinov and co-workers compared the synthesis of several aggregating and non-aggregating peptide syntheses using Fmoc- and Boc-chemistry SPPS. ${ }^{[36]}$ A linear relationship between the swelling volume changes and the molecular weight change of the resinbound peptide was determined for non-aggregating peptides, as previously also reported by Merrifield and co-workers. ${ }^{[33]}$ For aggregating peptides they observed significant shrinking of the resin. In 2019, Seeberger and co-workers reported on a similar reactor, albeit with an improved synthesis setup. ${ }^{[37]}$ Using parallel pressure- and UV-monitoring they experimentally validated a clear correlation between in-line UV analysis and volume changes of the resin upon aggregation.

\section{$2.4 \quad$ Instrumentation}


In addition to customized set ups described above, multiple flow-based synthesizers were commercialized in the early 1980s and the technology became accessible to a broader community notably all systems were based on Fmoc-chemistry SPPS. Details on these machines are described elsewhere and we will therefore only briefly describe timelines and technological advancements. ${ }^{[38]}$ The first two instruments, the PEPSYNthesizer I from Cambridge Research Biochemicals (CRB) and the Model 4175 from LKB Biochrom, came on the market in 1983. These synthesizers were manual instruments, although the potential for automation of flow-based SPPS was noticed early on. It was therefore no surprise, that CRB's first semiautomated system PEPSYNthesizer II was already commercialized two years later and served as the basis for the first fully automated 9020 PepSynthesizer by MilliGen. The total synthesis time per coupling and deprotection cycle was 57 minutes and the instrument was already able to synthesize up to three peptides in parallel. In the same year, LKB also developed an automated flow synthesizer (the Biolynx Model 4170), which was later sold by Novabiochem. In 1996, PerSeptive Biosystems commercialized the "Pioneer". The synthesis scale could be adjusted between 0.020 and $2.0 \mathrm{mmol}$ and amino acids were stored as dried powders before the activator (1-[bis(dimethylamino)methylene]-1H-1,2,3-triazolo[4,5-b]pyridinium 3-oxide hexafluorophosphate, HATU) and base (N,N-diisopropylethylamine, DIEA) were added prior to the coupling and recycling step.

\subsection{Summary and conclusions}

Flow-based SPPS was developed from the simple idea to develop "synthesis in columns",[17] to fully automated commercially available synthesizers, ${ }^{[38]}$ which allowed for in-line reaction monitoring and precise control of reagent parameters (Figure 2A). However, the instruments operated at room temperature, and used recycling loops to recirculate amino acid reagents until reaction completion. These designs conserved reagents, but missed other advantages of the flow approach, including the potential to: 1) achieve maximal reagent concentration within the solid support; 2) extend coupling time independently of activation time, by in-line activation; and 3) control heat transfer. In the next section, we discuss how some of these advantages are incorporated in flow-based instruments that utilize a 'preheat/activation loop' to facilitate SPPS at elevated temperature (Figure 2B). 
A) Erickson's flow peptide synthesizer

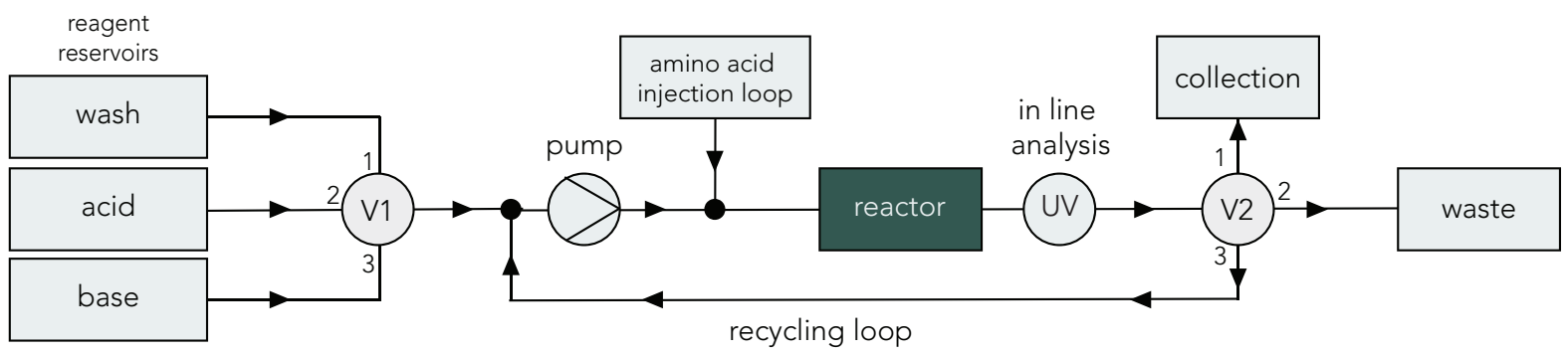

B) Pentelute's automated fast-flow peptide synthesizer

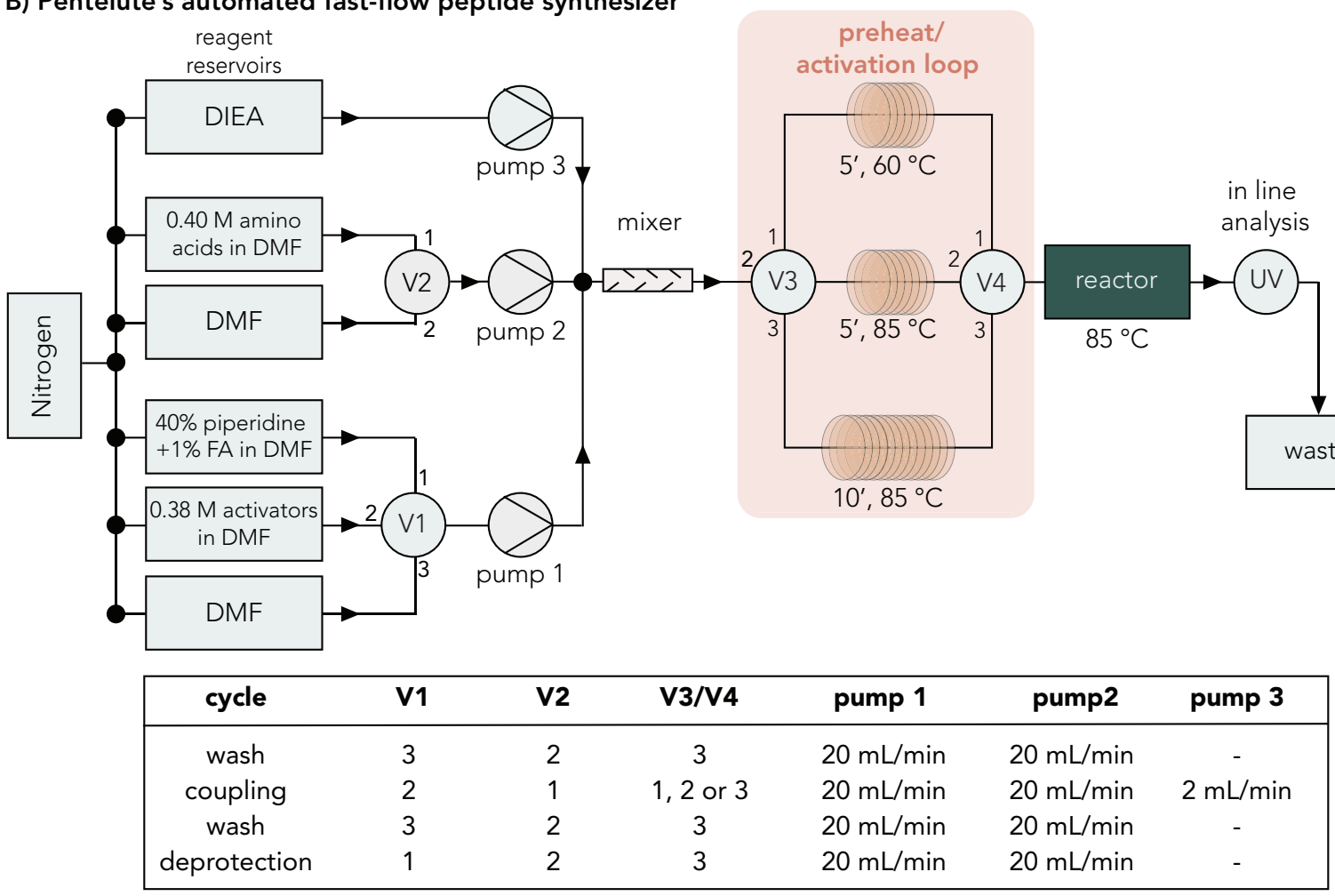

Figure 2 Automated synthesizer set ups. (A) Synthesis set up reported by Erickson and co-workers [21]. All reagents are delivered via a single pump, UV traces are recorded in-line and coupling solutions are circulated via a recycling loop; (B) Overview of the automated fast-flow peptide synthesizer (AFPS) build in the Pentelute group. Reagents are delivered via three pumps and activated in one of three heated preheat/activation loops and coupled in a heated reactor, UV traces are recorded in-line[12].

\section{Recent advances and developments}

\subsection{Flow-based SPPS utilizing a preheat loop}

In 2014, the Pentelute group reported on a manual 'fast-flow' SPPS protocol that significantly accelerated flow-based SPPS by means of a heated pre-heat loop and reactor. ${ }^{[13]}$ They reasoned that if amide bond formation under typical SPPS conditions is complete in less than 2 min and the reaction rate doubles for every $10{ }^{\circ} \mathrm{C}$ increase in temperature, the reaction should be completed in under $10 \mathrm{~s}$ at $60{ }^{\circ} \mathrm{C}$. Pentelute and co-workers therefore developed a manual flow chemistry set up that delivered 
reagents and solvents through a preheated activation loop to the heated reactor containing the solid support. While the activated ester, which is prone to by-product formation such as epimerization, is formed under highly controlled micro-flow conditions in the pre-heat loop, the resin is located in a tube-like reactor with a $1.27 \mathrm{~cm}$ inner diameter (total reactor volume $=2 \mathrm{~mL}$ ), which is in a meso-flow regime. This set up locally separates active ester formation and peptide coupling and therefore prevents by-product formation and degradation of reactive intermediates. ${ }^{[13,39]}$ With this procedure, coupling, deprotection and washing times were significantly shortened and a full cycle was completed within 3 min. As discussed below ('Related research areas and future directions'), the gain in speed achieved by 'fast-flow' synthesis is accompanied by an increase in reagent consumption (10 equivalents of amino acid per coupling cycle here, vs. 4-5 equivalents for manual synthesis). If coupling time is extended, flow rate increased, or the synthetic scale reduced (for example, when using solid supports of reduced loading), reagent excesses could be increased further.

Preheat/activation loops have since been employed in a number of other custom flow-based SPPS set ups. The Gordon group designed a "rudimentary system" with a similar design as the manual flow set up and screened key parameters such as resin matrices, temperatures, flow rates, coupling reagents, bases, and coupling solution concentrations with the goal of establishing a low cost system with minimal consumption of materials. ${ }^{[40]}$ In addition, Seeberger and co-workers built a similar flow-based synthesizer and included a variable-bed reactor (described above). ${ }^{[37]}$

\subsection{Automated flow-based SPPS (AFPS)}

Based on their manual flow set up, Pentelute and co-workers developed an automated 'fast-flow' peptide synthesizer (AFPS), which showed significant improvements with respect to temperature control, fluid handling and reproducibility. Briefly, the system contains three High-performance liquid chromatography (HPLC) pumps (Figure 2B): pump 1 (20 mL/min) is assigned to coupling agents, deprotection solution and solvent. An identical pump (pump 2) is dedicated to amino acid stock solutions and solvent. These two pumps are connected to multiple stock solutions and can be selected via a software-controlled system of valves (represented by V1 and V2). In addition, pump 3 ( $2 \mathrm{~mL} / \mathrm{min}$ ) is dedicated to the coupling base and is only pumping reagent during the coupling step. All solutions are pumped through tubing of equal length to a fluid mixing valve. After passing a mixing loop, activation takes place in one of three heated activation loops. The solution is then passed through the resin in a disposable PEG syringe, which is embedded in a heated reactor. The fluid is monitored via an in-line UV-Vis detector before it is discarded. The entire system is controlled via LabView software and allows for easy monitoring of all critical parameters (e.g. pressure and temperature). Synthesis data including time-resolved Fmoc-deprotection data (from the UV-Vis detector) is collected and stored during every synthesis step. In contrast to previous flow-based peptide synthesizers (Figure 2A), this set up contains two major modifications: (a) three pre-heated activation loops, which allow for tailoring of the activation conditions, (b) a disposable, small volume reaction vessel which allows for simplified handling of the resin and allows for rapid addition and removal of all reagents.

Automation and additional increase in temperature further accelerated the process and enabled the synthesis of a $30 \mathrm{mer}$ in less than 30 minutes of synthesis time. Multiple peptides in the size range of 
30 amino acids were synthesized and crude peptide purities and yields were comparable to standard batch SPPS. Coupling of cysteine and histidine, two amino acids at a particularly high risk for epimerization, was thoroughly investigated and optimized. The content of D-amino acid was dependent on the protecting group, temperature and flow rate (and therefore the activation time) and independent of coupling agent (HATU/(7-azabenzotriazol-1-yloxy)trispyrrolidinophosphonium hexafluorophosphate, PyAOP) and total coupling cycles. ${ }^{[12,14]}$ Activation of Fmoc-Cys(Trt)-OH and Fmoc-His(Boc)-OH with PyAOP at $60{ }^{\circ} \mathrm{C}$ through a shorter 5' preheat-activation loop resulted in minimal D-amino acid content. In addition, synthesis on the AFPS showed an improvement for the synthesis of "difficult peptides", which can likely be attributed to the high synthesis temperature. In this initial report, the Pentelute group demonstrated that their AFPS enables remarkably fast synthesis of peptides with equal quality as batch and microwave procedures. However, the question if AFPS may be optimized to deliver longer peptide sequences remained unaddressed.

\subsection{Optimized AFPS}

Initially, AFPS was developed with the aim to accelerate peptide synthesis. ${ }^{[12,13]}$ More recently, the resulting gains in speed were leveraged to optimize synthetic protocols. ${ }^{[14]}$ This process was carried out in stages - first by replicating syntheses of glucagon-like peptide-1 (GLP-1) and growth hormonereleasing hormone $(\mathrm{GHRH})$ peptides, using crude product profiles as readout—and then by synthesis of a wider range of peptides, using in-line UV-Vis monitoring to infer coupling yields obtained under different conditions. Some improvements suggested by the first round of experiments are unique to AFPS, such as the use of reduced flow rates to minimize pump cavitation, and the use of $90{ }^{\circ} \mathrm{C}$ for reagent activation in the 'preheat/activation' loop. Others are more generally applicable, and include the use of high-purity reagents, high concentrations of amino acid active esters for coupling, and 'buffered' piperidine solutions for Fmoc removal ('Optimized AFPS in context', below).

Perhaps most interesting are the amino acid-specific coupling conditions suggested by the study of hundreds of individual coupling yields. As discussed below ('Optimized AFPS in context'), accumulating data support the notion of residue-specific coupling efficiencies in AFPS, and the effectiveness of residue-specific conditions to minimize them. Amino acid-specific conditions suggested by the original work include: extended coupling times for Fmoc-Ser $(\mathrm{tBu})$ and Fmoc-Ala, and extended coupling time combined with PyAOP activation for Fmoc-Cys(Trt), Fmoc-His(Boc), Fmoc-Asn(Trt), Fmoc-Gln(Trt), Fmoc-Arg(Pbf), Fmoc-Thr(tBu), and Fmoc-Val. The combined effect of optimized parameters on the synthesis of GLP-1 is shown in Figure 3. 


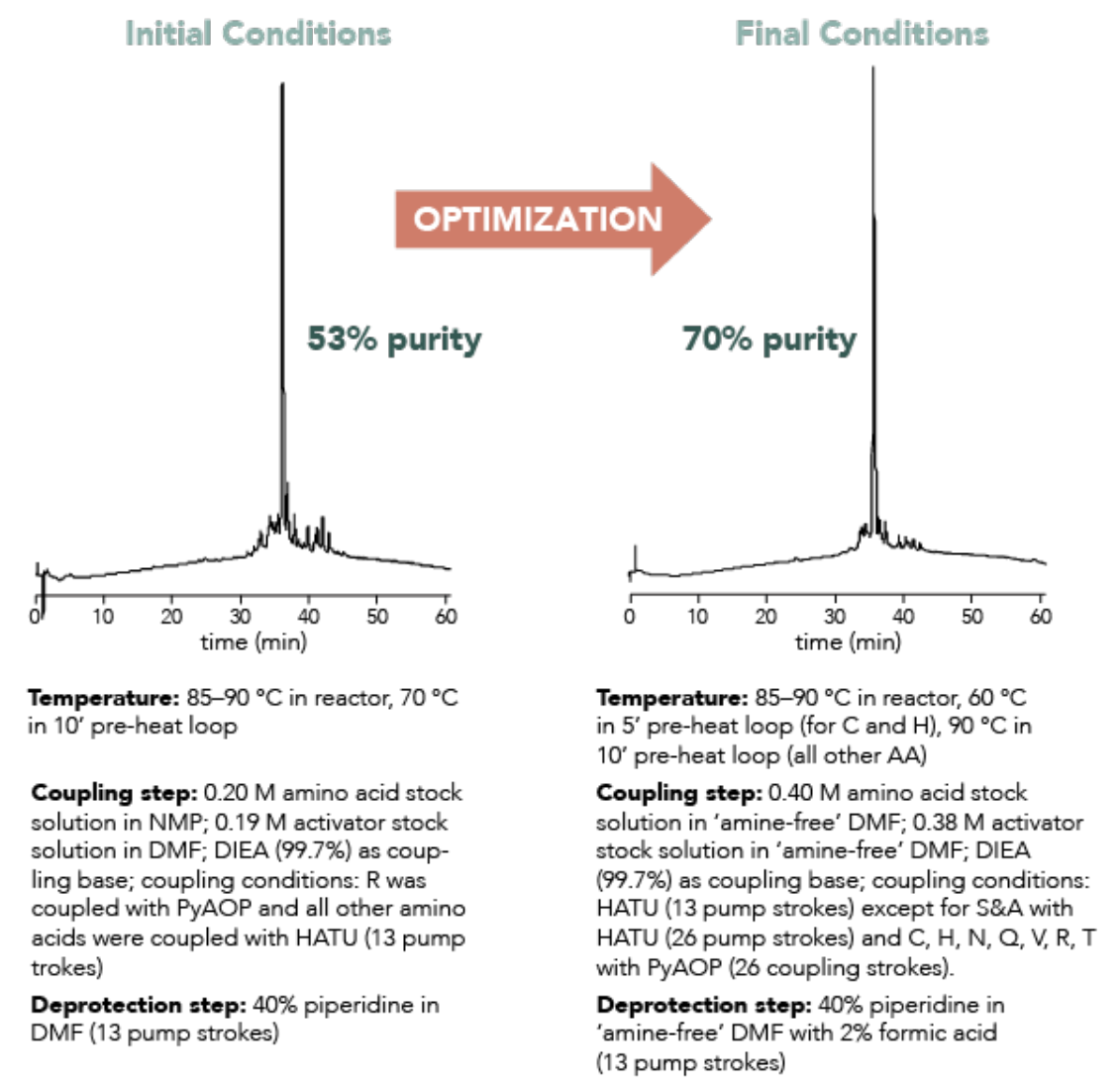

Figure 3 Automated fast-flow peptide synthesis optimization with respect to chain length and total synthesis time. Depicted are the starting point and the end point for the optimization process, here showcased for GLP-1 (30 amino acids). All concentrations are given for the stock solutions and are not final concentrations. Syntheses on the AFPS are performed on $\sim 0.10-0.02 \mathrm{mmol}$ scale. All stock solutions are diluted according to figure 2. Reproduced with modifications from [14]. From N. Hartrampf, A. Saebi, M. Poskus, Z. P. Gates, A. J. Callahan, A. E. Cowfer, S. Hanna, S. Antilla, C. K. Schissel, A. J. Quartararo, X. Ye, A. J. Mijalis, M. D. Simon, A. Loas, S. Liu, C. Jessen, T. E. Nielsen, B. L. Pentelute, Science 2020, 368, 980-987 [14]. Modified with permission from the corresponding author, reprinted with permission from AAAS.

\subsection{Protein Synthesis by optimized AFPS}

Could an optimized flow-based protocol extend the general utility of stepwise synthesis for accessing full-length protein chains? Full-length proteins have occasionally been prepared by stepwise SPPS, including flow-based SPPS (for selected examples, see references [41-47]), and the resulting synthetic products have proven useful for structure-function and high-resolution structural studies. ${ }^{[48,49]}$ However, expert opinion holds that stepwise synthesis is not an effective means of preparing peptides beyond 40-50 residues in length as single, defined covalent species. ${ }^{[4,5]}$ This limitation is due to the statistical accumulation of low-level co-products that occurs even with optimized protocols $(<0.1 \%$ side products/cycle; 'Optimized AFPS in context', below), and is what motivated the development of chemical ligation as a means to access synthetic proteins in homogeneous form.

Recently, we tested the optimized AFPS protocol in the synthesis of nine protein chains. The synthetic targets spanned 86-164 amino acids in length and included historically significant targets such as 
human proinsulin, the human immunodeficiency virus-1 (HIV-1) protease, and human lysozyme. ${ }^{[14]}$ The homogeneity of crude synthetic products varied, but in general show that AFPS furnishes synthetic peptides in higher purity than conventional Fmoc SPPS, on a considerably faster timescale ( $\sim 2.5 \mathrm{~min} /$ cycle here vs. $\sim 1 \mathrm{~h} /$ cycle standard). Analytical data for crude HIV-1 protease are shown in Figure 4. A sequence nearly identical to one previously studied by stepwise synthesis gave considerably cleaner crude material, qualitatively similar to that obtained by optimized Boc SPPS ${ }^{[50]}$. With the gains in speed achieved by AFPS, it should be feasible to significantly expand empirical data for syntheses of longer chains, and to define in more quantitative terms the outcomes of SPPS near its practical 'upper bound' (for example, as a distribution of crude analytical purities vs. chain length).

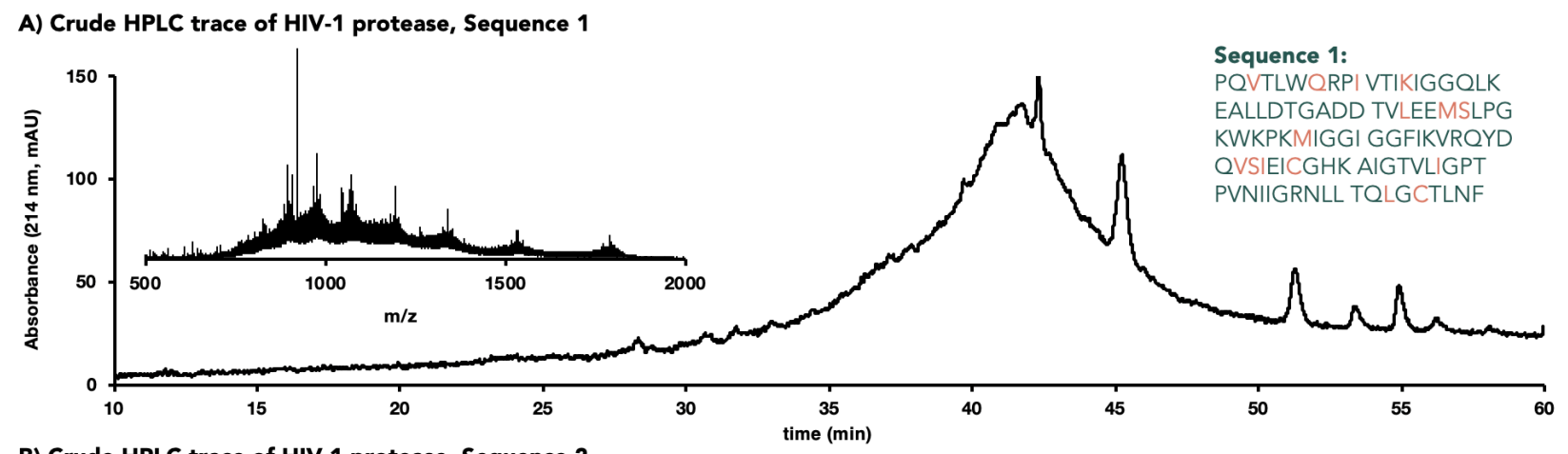

B) Crude HPLC trace of HIV-1 protease, Sequence 2

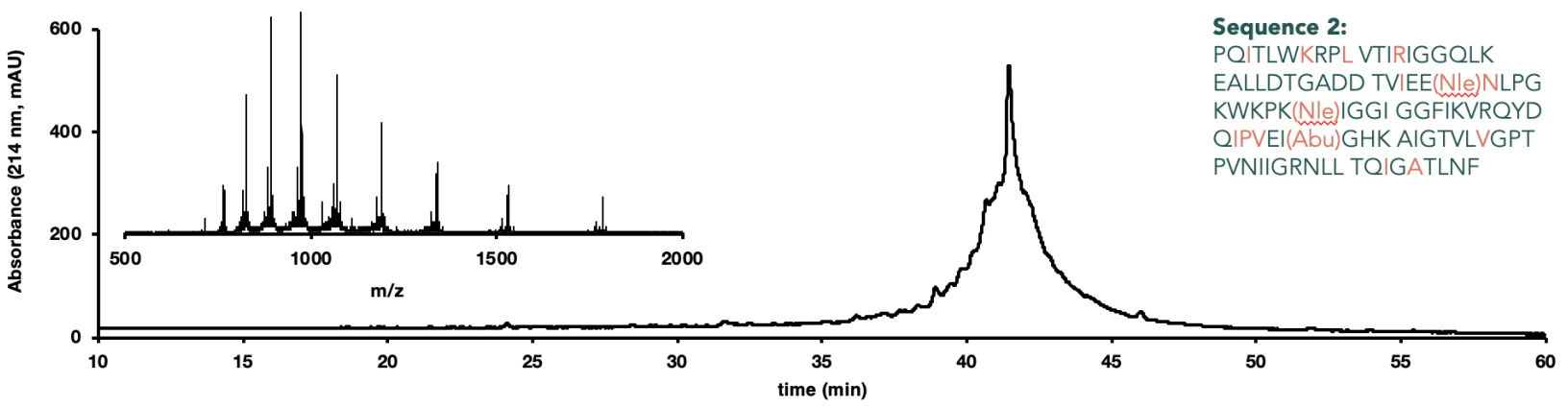

Figure 4 Crude analytical HPLC traces of two HIV-1 proteases prepared by AFPS. A) HIV-1 protease, from Servais et al. [15] B) HIV-1 protease, from Johnson et al. [16], based on a sequence previously accessed by stepwise Boc synthesis [38f] The two sequences have $85 \%$ similarity. Reproduced with modifications from the supporting information of [14]. 'Nle' denotes norleucine; 'Abu' denotes 2-aminobutyric acid. MS spectra were integrated over the entire peak area. From N. Hartrampf, A. Saebi, M. Poskus, Z. P. Gates, A. J. Callahan, A. E. Cowfer, S. Hanna, S. Antilla, C. K. Schissel, A. J. Quartararo, X. Ye, A. J. Mijalis, M. D. Simon, A. Loas, S. Liu, C. Jessen, T. E. Nielsen, B. L. Pentelute, Science 2020, 368, 980-987 [14]. Modified with permission from the corresponding author, reprinted with permission from AAAS.

AFPS could considerably reduce the time and labor associated with chemical protein synthesis, either by furnishing full-length protein chains directly, or by facilitating the synthesis of peptide segments for use in chemical ligation. In general, we believe that stepwise synthesis and chemical ligation can be viewed as complimentary approaches to the chemical synthesis of small proteins. Chemical ligation is inherently convergent, and in principle more efficient with respect to material yield, whereas stepwise synthesis avoids intermediate isolation steps and is thus more efficient with respect to time. Likewise, chemical ligation provides the opportunity for analytical characterization and purification at the stage 
of peptide segments, whereas for stepwise synthesis, characterization and purification are performed on the final product only. Depending on one's application, one or the other approach may be more appropriate. For any work in which chemical protein synthesis is a significant focus, best practices for the characterization of synthetic products should be followed. ${ }^{[51]}$

Two independent syntheses of barnase, carried out in the same laboratory, enable a comparison of the linear and convergent strategies in practice. ${ }^{[14,52]}$ The convergent approach involved chemical ligation of four peptide segments (Figure 5A). For each segment, manual flow-based synthesis was carefully optimized with respect to coupling agents, backbone protecting groups and resin. In contrast, linear synthesis by AFPS gave the desired product directly, using the generic optimized procedure (Figure 5B). The two routes yielded comparable amounts of full-length barnase samples $(3 \%$ for the ligation route, starting from crude peptide segments; $2 \%$ for the stepwise route), which upon refolding exhibited similar activities relative to recombinant barnase ( $89 \%$ vs. $84 \%$ for the ligation vs. stepwise routes, respectively). These results parallel a comparison between ligation and stepwise SPP approaches to the 51-residue turkey ovomucoid third domain, ${ }^{[53]}$ and suggest that stepwise synthesis may be a viable route to small proteins of adequate purity for many applications. Additional comparative studies of this type could help to clarify possible material differences in synthetic protein samples obtained by the two approaches, and would ideally employ an even wider range of analytical techniques. 


\section{A) Convergent approach}

Ligation of 30-50 amino acid fragments synthesized by manual flow chemistry

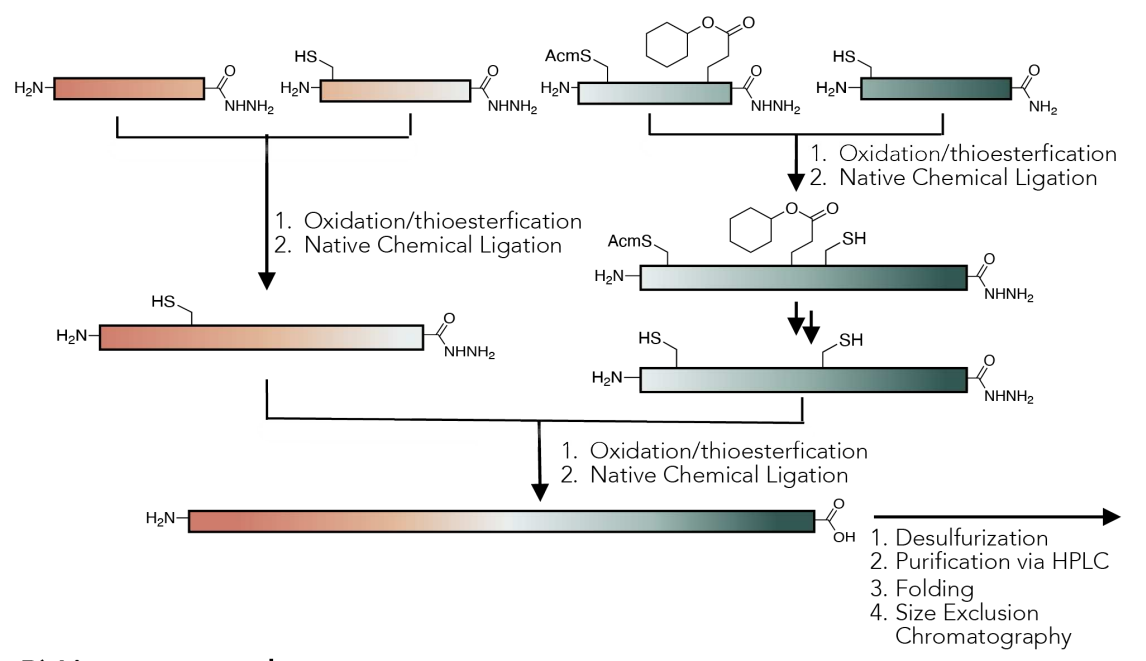

B) Linear approach

\author{
Features: \\ - individually-optimized \\ strategies \\ - labor-intensive/ \\ time consuming \\ - modular \\ - characterize/purify \\ intermediate, segments'
}
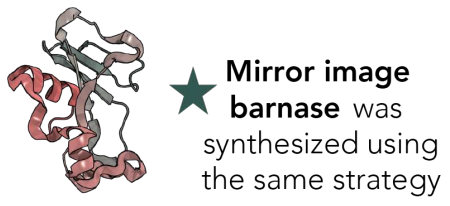

barnase

Stepwise assembly of $>50$ amino acid peptides using automated flow peptide synthesis (AFPS)

$\mathrm{H}_{2} \mathrm{~N}-$

barnase with non canonical p-bromophenylalanine for future

derivatization was synthesized using the same strategy

\section{Examples synthesized:}

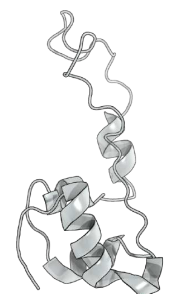

Proinsulin

86 AA, $3.5 \mathrm{~h}$

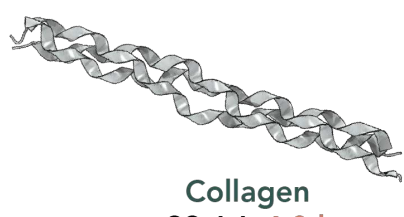

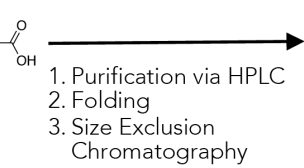

Chromatography

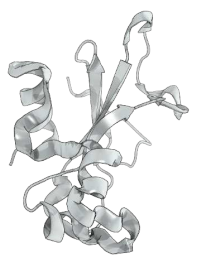

Barnase

$110 \mathrm{AA}, 4.5 \mathrm{~h}$

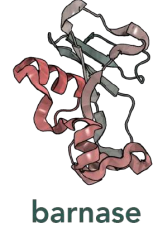

barnase
Features:

- general ,recipe'

- rapid

- non-modular

- characterize/purify

final product only

synthesis time $=4.5 \mathrm{~h}$

(total production time: 44-56 h)

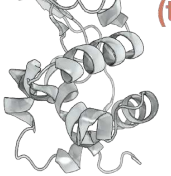

Lysozyme 129 AA, 5.5 h

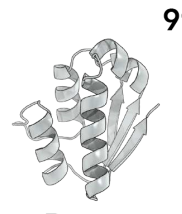

Barstar $90 \mathrm{AA}, 3.5 \mathrm{~h}$

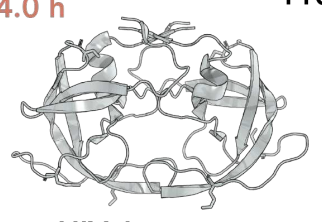

HIV-1 protease

99 AA, $4.5 \mathrm{~h}$

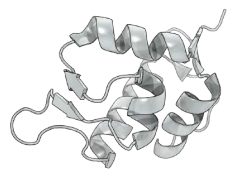

$\operatorname{MDM} 2^{[1-118]}$

$127 \mathrm{AA}, 5.0 \mathrm{~h}$

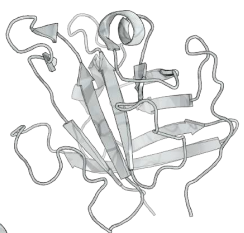

Sortase $A^{*}$ $164 \mathrm{AA}, 6.5 \mathrm{~h}$

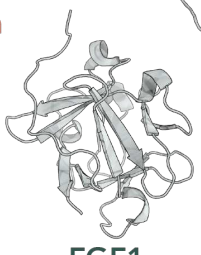

FGF1

$140 \mathrm{AA}, 5.7 \mathrm{~h}$

Length of amino acid sequences

Figure 5 Comparison between a convergent and a linear approach for the flow-based synthesis of barnase (110 AA). A) barnase was prepared from four fragments using three ligation steps and four additional chemical manipulations. Reproduced with modifications from [41]. B) barnase was synthesized using a stepwise approach. No additional chemical manipulations or ligations were required. Additional protein examples, which were synthesized by the same approach, are depicted below - along with their synthesis times [41].

\section{$4 \quad$ Optimized flow-based Fmoc SPPS in context}

Historically, SPPS required significant optimization to reach its potential. ${ }^{[54]}$ In this section, we briefly discuss lessons learned from this broader context, and their relevance to optimization of flow-based 
Fmoc SPPS, which we discuss in greater depth. In parallel, we show how rapid flow-based synthesis is already generating new data that supports and extends our understanding of SPPS more generally, and could drive further progress-both for specialized flow-based instruments, and more conventional practice.

\subsection{Optimized Boc SPPS}

Prior to the 1980s, chronic side reactions limited the scope of Boc-chemistry SPPS to chains of $\sim 30$ residues or smaller. These side reactions-including trifluoroacetylation, serial residue 'deletions', and chain termination-accounted for 4-5\% per cycle, occurred independently of sequence, and were reduced to less than $0.1 \%$ by the use of optimized protocols. ${ }^{[50,55-57]}$ Minimization or elimination of chronic side reactions, along with the identification of dimethylformamide and neat trifluoroacetic acid as effective solvents for reducing the occurrence of 'difficult couplings' (below), resulted in a highly effective Boc-chemistry SPPS protocol. In ideal cases, optimized stepwise Boc-chemistry SPPS could be used to prepare peptides corresponding to single-domain protein molecules, such as HIV-1 protease. ${ }^{[58]}$

Many of the lessons learned from the successful optimization of Boc-chemistry SPPS apply equally well to the Fmoc approach, including flow-based Fmoc SPPS. These lessons include the use of solid support resins free of extraneous chemical functionalities, high concentrations of amino acid active esters during coupling, high-purity reagents, and DMF as solvent. As expected, many of these considerations improved the performance of automated flow-based Fmoc-SPPS. ${ }^{[14]}$ In addition, Fmocchemistry SPPS presents challenges not common to Boc-chemistry. These include the formation of aspartimide side products during Fmoc deprotection by piperidine, and the exacerbation of 'difficult couplings', which would be otherwise minimized by use of trifluoroacetic acid in Boc-chemistry SPPS.

\section{2 'Difficult couplings'}

Assuming that chronic side reactions have been eliminated, sequence-dependent incomplete peptide bond-forming reactions_-so-called 'difficult couplings' - are the greatest impediment to high-fidelity SPPS. Non-random 'difficult couplings' generally occur 10-15 residues from the protected peptide Cterminus, occur for roughly one out of five peptides, and are defined by a series of incomplete coupling reactions which are not significantly affected by repeat coupling treatments. ${ }^{[59]}$ The proposal that 'difficult couplings' are caused at the molecular level by the formation of intermolecular H-bonded aggregates ${ }^{[57,59,60]}$ is generally accepted. ${ }^{[61]}$ However, since the original work, the basic empirical observations surrounding 'difficult couplings' have not been systematically expanded.

Accelerated SPPS provides an opportunity to examine the occurrence of 'difficult couplings' during the synthesis of a larger number of peptides than would otherwise be practical, and to refine our understanding of the phenomenon. In Figure $\mathbf{6}$ we illustrate the onset of 'difficult couplings' encountered during AFPS of nearly 400 distinct 20-30 residue peptides, documented in recent work. ${ }^{[62]}$ 'Difficult couplings' were identified by spectroscopic monitoring of Fmoc deprotection, considering both the integrated UV absorbance (proportional to the yield of the previous coupling) and the UV peak width (which increases upon chain aggregation). 
Based on the spectroscopic definition, 'difficult couplings' occurred with greater frequency than documented previously ( $\sim 50 \%$ of 20 -mer peptides $^{[62]}$, vs. $\sim 20 \%$ previously ${ }^{[59]}$ ). This difference may reflect a more sensitive definition of aggregation in the recent work, or a bias in the new dataset toward difficult peptides, since the dataset consisted of peptides from a limited number of projects. The data reproduced to some extent the maximal occurrence of 'difficult couplings' at 10-15 residues from the C-terminus. However, in contrast to the original data, they also show that 'difficult couplings' occur with significant frequency at all chain lengths. Further work is needed to identify the origin of differences between these and earlier observations (for example, Fmoc-chemistry SPPS/dibenzofulvene monitoring/elevated temperature recently vs. Boc-chemistry SPPS/ninhydrin monitoring/ambient temperature previously). Important future objectives will include correlating the 'difficult couplings' inferred from spectroscopic data with precise coupling yields (for example, from quantitative ninhydrin assay or LC-MS analysis of crude peptide products), establishing the effect of temperature on the onset and severity of 'difficult couplings', and documenting the prevalence of 'difficult couplings' in Fmoc SPPS using even larger datasets.
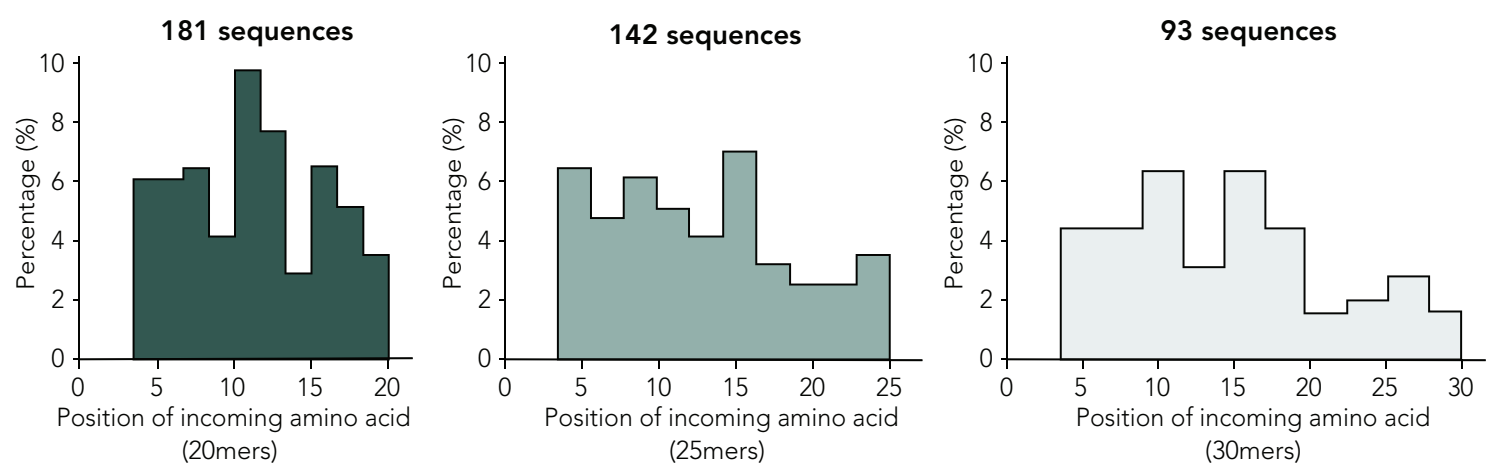

Figure 6 Onset of aggregation during AFPS of 20-30 residue 'difficult' peptides, grouped according to length.

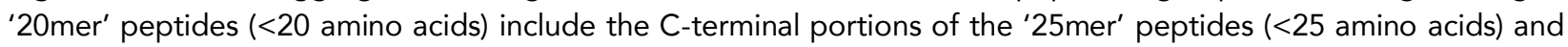
'30mer' peptides; '25mer' peptides include the C-terminal portions of the '30mer' peptides. For each group, sequences that exhibited an aggregation event were binned according to the onset of aggregation, and normalized to the total aggregating sequences within that group. Aggregation was inferred by a change in two sequential dibenzofulvene signals (where the normalized difference between the height and the width changed $>20 \%$ ). Position is defined relative to the peptide C-terminus. Reproduced with modifications from https://doi.org/10.26434/chemrxiv.12649343.v1 [62] with permission of the corresponding author (originally published on ChemRxiv under CC BY-NC-ND 4.0 license).

\subsection{Elevated temperature to facilitate 'difficult couplings'}

Many strategies intended to disrupt the formation of interchain hydrogen bonds have been employed to mitigate 'difficult couplings' and improve the outcomes of SPPS. Broadly, these strategies aim to block the formation of aggregate structures either directly-for example, by reversible protection of backbone $\mathrm{N}-\mathrm{H}$ groups — or indirectly, by the use of conditions that favor solvation of the protected peptide chain. Among the latter strategies, and one of the earliest approaches to facilitate 'difficult couplings', is the use of elevated temperature. ${ }^{[63-65]}$ 
In contrast to backbone protection, which requires the availability of distinct protected amino acid building blocks, elevated temperature is a potentially general solution that would apply equally well to a range of non-canonical peptides (for example, D-peptides). The most significant drawbacks to use of elevated temperature in SPPS are the generation of activated amino acids under poorly defined conditions (for example, microwave heating), and the tendency of heating to exacerbate epimerization of some amino acid active esters (especially cysteine derivatives, and histidine derivatives with nonelectron-withdrawing imidazole protection). As discussed elsewhere, ${ }^{[66]}$ AFPS is a potential solution to these challenges.

More work is needed to understand the full potential of elevated temperature in facilitating 'difficult couplings', and its utility in relation to other strategies. Here, automated flow-based SPPS is poised to make strong contributions - both to fundamental understanding, and to the development of improved tactics. For example, it would be interesting to see how the frequency and chain length-dependence of difficult couplings change as a function of temperature, when studied using large datasets. Does temperature serve to delay the onset of aggregation in susceptible peptides, or to reduce the severity of aggregation at specific positions only? It would also be interesting to study the effect of temperature in conjunction with backbone protection. Does backbone protection tend to lessen the beneficial effect of temperature, or are additive effects obtained? Some evidence suggests that backbone protection provides a greater benefit, ${ }^{[67]}$ but further work is needed to generalize these observations. Systematic comparisons between 'salvage' tactics could inform both further refinements in flow-based SPPS, as well as optimal manual SPPS strategies where specialized instrumentation is unavailable.

\subsection{Amino acid-specific coupling yields}

'Difficult couplings' are sequence-dependent, and not residue-specific per se. However, differences in the relative reactivity of activated amino acids ${ }^{[68]}$ may exacerbate otherwise slow coupling reactions. In the 1980s, yields for hundreds of coupling reactions were determined by quantitative ninhydrin assay, in both Boc- (Mixed anhydrides; 35 peptides) ${ }^{[59]}$ and Fmoc-chemistry SPPS (1-hydroxybenzotriazole, HOBt esters; 64 peptides). ${ }^{[69]}$ These data showed some agreement, with the coupling of valine, leucine, and isoleucine being frequently incomplete in both schemes $(\sim 20 \%$ of couplings in Boc-chemistry SPPS, and $\sim 40 \%$ in Fmoc-chemistry SPPS). However, differences were also apparent. For example, threonine-frequently incomplete in the Boc scheme (19\% of couplings)—was not especially problematic in the Fmoc scheme. Additionally, alanine couplings-rarely incomplete in Boc chemistry (4\% of couplings) — were among the most problematic in Fmoc chemistry ( $40 \%$ of couplings). In general, optimized SPPS protocols do not call for residue-specific conditions, perhaps because a consensus for amino acid-specific coupling yields has not been found.

AFPS provides an opportunity to re-evaluate residue-specific coupling efficiencies using larger datasets. With the goal of optimizing coupling conditions for flow-based SPPS, individual coupling yields were determined during SPPS of four different peptide test sets (each comprising $\sim 20$ distinct 30-mers), using four different protocols. ${ }^{[14]}$ Coupling yield was taken as the integrated UV signal generated by deprotection of the incorporated amino acid, divided by the signal from the prior residue. 'Yields' obtained in this way were intended to inform on relative trends over many measurements, 
rather than precise values of residual, unreacted amine (such as those obtained by quantitative ninhydrin assay).

Under conditions of HATU activation, the following amino acids had the lowest average coupling yields: Fmoc-Ala, Fmoc-Ser(t-Bu), Fmoc-Thr(t-Bu), Fmoc-Val, Fmoc-Gln(Trt), Fmoc-Asn(Trt), and Fmoc-Arg(Pbf). Of these, only Fmoc-Arg is readily explained by an established chemical mechanism. ${ }^{[0]}$ Similar trends were observed in a larger dataset, comprising $\sim 1520$ unique peptides synthesized on three separate instruments by multiple users. ${ }^{[62]}$ Figure 7A shows the distributions of coupling yields obtained for Fmoc-Asn(Trt) and Fmoc-Leu in the larger dataset, illustrating the greater proportion of incomplete couplings observed for Fmoc-Asn(Trt). In contrast, no significant difference was observed between the average coupling yields of Fmoc-Leu and a second 'non-problematic' amino acid (Fmoc-Met; Figure 7B). It will be interesting to see whether the same trends hold on even larger sets of peptides obtained by individual users, to minimize the possible influence of peptide sequence effects under optimally controlled experimental conditions. However, the data obtained to date appear to support the existence of significant amino acid-specific differences in coupling efficiencies, at least in flow-based SPPS.
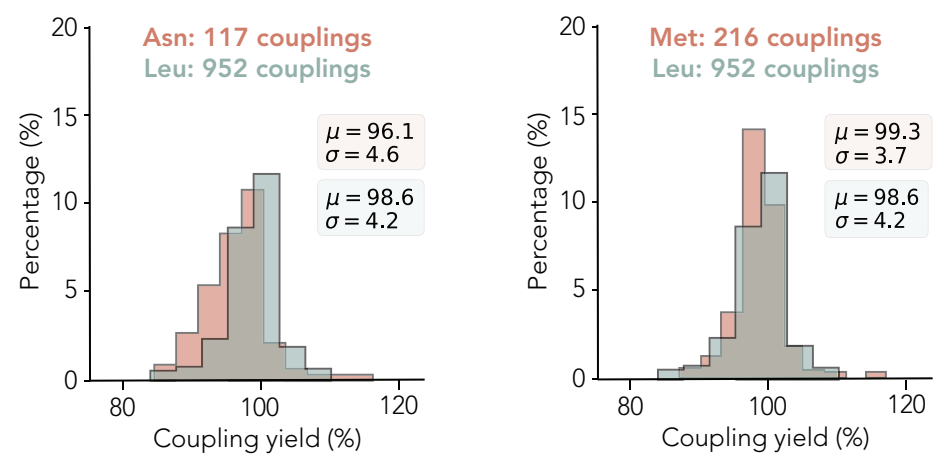

Figure 7 Overlaid coupling yield distributions, illustrating the lower average yields obtained for some amino acids (HATU activation). A) Distributions obtained for FmocAsn(Trt) (red) and Fmoc-Leu (green), illustrating the significant proportion of incomplete couplings in the case of Fmoc-Asn(Trt) only. B) Distributions obtained for Fmoc-Met (red) and Fmoc-Leu (green), illustrating two examples with high coupling yields. The yield for incorporation of an Fmoc-amino acid was inferred by integration of the dibenzofulvene signal generated by its deprotection, normalized to the integrated signal from the preceeding residue. This procedure accounts for the downward trend in dibenzofulvene signals generally observed over a synthesis, but can result in yields in excess of $100 \%$. Reproduced with modifications from https://doi.org/10.26434/chemrxiv.12649343.v1 [62] with permission of the corresponding author (originally published on ChemRxiv under CC BY-NC-ND 4.0 license).

\subsection{Amino acid-specific coupling conditions}

Minimizing residue-specific differences in coupling yield provided a framework for optimizing flowbased SPPS. During this process, it was observed that incomplete couplings of problematic residues such as Fmoc-Asn(Trt) were less frequent under conditions of PyAOP activation (a combination of PyAOP and extended coupling duration). ${ }^{[14]}$ Reduction in incomplete couplings of frequently- 
problematic residues by PyAOP activation suggested the use of amino acid-specific coupling conditions in flow-based SPPS (a 'recipe'), where PyAOP activation was used for frequentlyproblematic residues only.

The effect of PyAOP activation was maintained when studied over a larger number of syntheses. ${ }^{[62]}$ As for the smaller dataset, the data for each coupling condition were obtained on different peptides, rather than by replicate syntheses under each condition. Frequently problematic residues (e.g., FmocAsn(Trt)) showed different coupling yield distributions under conditions of HATU vs. PyAOP activation (Figure 8A), whereas non-problematic residues (e.g. Fmoc-Leu) showed similar distributions under both sets of conditions (Figure 8B). It will not be surprising that choice of coupling agent might critically impact a synthesis. Nevertheless, large-scale comparative studies of activation conditions in SPPS are critically lacking, ${ }^{[11,72]}$ and this is an area where automated synthesis could have a major impact. By studying the effect of a reaction parameter on hundreds or thousands of coupling reactions rather than just a handful, better predictive understanding of its likely effect in new contexts might be obtained. Further work along these lines would ideally study the effect of single varied parameters on replicate syntheses within a single test set, to unambiguously demonstrate the causal effect of the varied parameter.
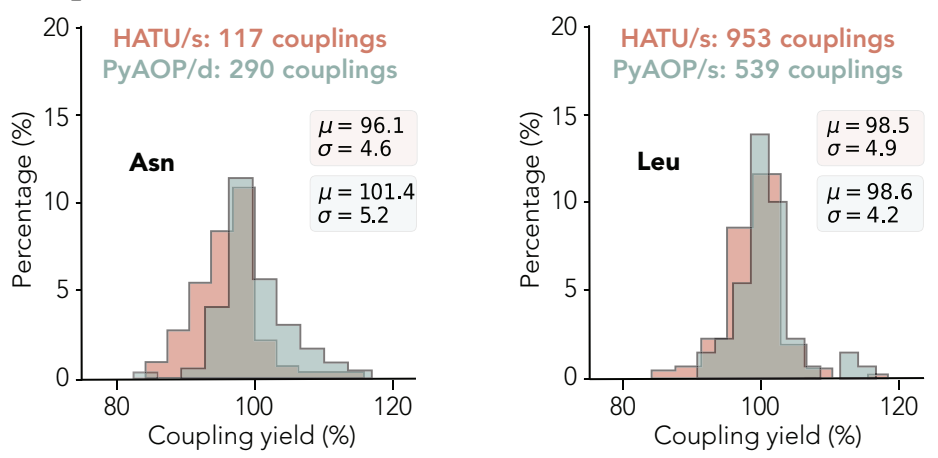

Figure 8 Overlaid coupling yield distributions, illustrating the effect of conditions on the coupling of either (a) Fmoc-Asn(Trt) or (b) Fmoc-Leu. Differences in coupling yields were obtained for the problematic Fmoc-Asn(Trt) only. $s=$ normal coupling time, $d=$ extended coupling time. Reproduced with modifications from https://doi.org/10.26434/chemrxiv.12649343.v1 [62] with permission of the corresponding author (originally published on ChemRxiv under CC BY-NC-ND 4.0 license).

Another interesting observation on coupling conditions is shown in Figure 9, which illustrates the effect of coupling duration on reaction yield. 'Coupling time' has a unique meaning in flow-based SPPS, since 'activation time' (the time an Fmoc-Xaa-OH and activating agent are combined with base) remains constant, even as the coupling time is extended. ${ }^{[62]}$ This is in contrast to a batch process, where extended coupling occurs concomitant with extended activation. Therefore, extended couplings in flow-based SPPS are analogous to 'multiple couplings' in batch synthesis, where fresh coupling solution is added after an initial treatment, in an effort to increase reaction yield. Figure 9A shows that for couplings of Fmoc-Asn(Trt), no benefit was conferred by extended coupling time, consistent with the observation that 'difficult couplings' are not significantly improved by 'multiple coupling' treatments in batch SPPS. ${ }^{[56]}$ In contrast, couplings of Fmoc-Ala exhibit a small but significant benefit 
from extended duration (Figure 9B). Further work is needed to re-evaluate the utility of 'multiple couplings' in the context of flow-based synthesis, and to understand the mechanistic origin of contextdependency.
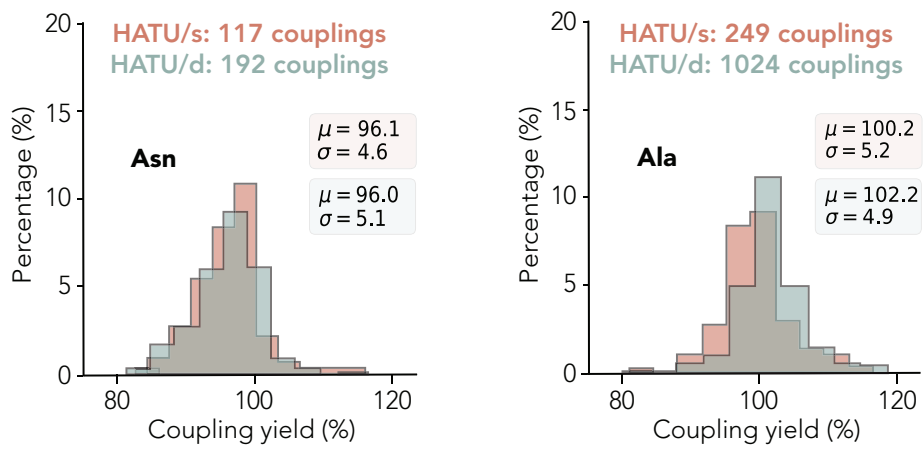

Figure 9 Overlaid coupling yield distributions, illustrating the effect of coupling time on yields for (a) Fmoc-Asn(Trt)-OH or (b) Fmoc-Ala-OH couplings. FmocAsn(Trt) couplings were not improved by extended time, whereas Fmoc-Ala couplings exhibited a small but significant benefit. $s=$ normal coupling time, $d=$ extended coupling time. Reproduced with modifications from https://doi.org/10.26434/chemrxiv.12649343.v1 [62] with permission of the corresponding author (originally published on ChemRxiv under CC BY-NC-ND 4.0 license).

\subsection{Minimization of aspartimide formation}

Preventing aspartimide formation in Fmoc-chemistry SPPS remains a topic of great interest. Aspartimide formation is exacerbated by elevated temperatures, which are otherwise beneficial for peptide synthesis. Many strategies to suppress aspartimide formation while keeping the synthesis temperature high were therefore investigated. These can be broadly divided into three classes: (a) new aspartic acid protecting groups, (b) backbone protection and (c) modifications of the deprotection base. For aspartic acid protecting groups it was generally found that bulky esters reduce aspartimide formation, ${ }^{[73]}$ and recently Bode and co-workers reported on the use of cyanosulfurylides as effective protecting groups. ${ }^{[74]}$ Blocking the amide backbone of aspartic acid is the most effective strategy to avoid aspartimide formation, however, coupling to secondary amines is more difficult and backboneprotected dimeric building blocks are expensive. Either lowering the $\mathrm{pK}_{\mathrm{A}}$ of the deprotection base (e.g. by the use of piperazine) or addition of acids (e.g. formic acid, HOBt and 4-nitrophenol) to the deprotection base (in most cases piperidine in DMF) are a third popular strategy. ${ }^{[75]}$

In flow chemistry, addition of formic acid to the deprotection base is nearly as effective as backbone protection at minimizing aspartimide formation, even at elevated temperature. In their optimization studies, Pentelute and co-workers screened many of the aforementioned strategies to minimize aspartimide formation in flow. ${ }^{[14]}$ Bulky protecting groups only had a limited effect, whereas addition of $1 \%$ formic acid to a $20 \%$ piperidine solution in DMF was sufficient to minimize aspartimide formation in most sequences. In some Asp-Gly-containing peptides, such as collagen, 2, 4dimethoxybenzyl (DMB)-protected glycine was used to further reduce aspartimide formation. Notably, 
Asp-coupling to the DMB-protected secondary amine was completed within seconds, thereby rendering backbone protection in flow more practical than by traditional methods.

\section{$5 \quad$ Related research areas and future directions}

Flow-based solid phase peptide synthesis is regaining momentum as it presents a unique opportunity for in-line data collection, minimized by-product formation and exceptionally fast synthesis on solid support. Several milestones have led to the technology in its current state: the introduction of FmocSPPS to enable time-resolved UV-Vis monitoring, and the introduction of a preheat loop for the fast activation of amino acid building blocks. Optimization of AFPS aimed at either decreasing synthesis time or improving crude product quality. However, general challenges associated with peptide synthesis, such as toxicity of reagents and atom economy, remain unaddressed. In the following paragraphs we will therefore highlight several active and related research areas with potential impact on AFPS. We will only focus on solid-phase synthesis methods in this section although significant achievements also were made in solution phase peptide synthesis.

\subsection{Technological advancements}

Developments in flow-based synthesis have tremendously benefitted from interdisciplinary research and technological advancements, such as improved equipment, faster computers and new technology for data analysis. Flow chemistry itself is becoming increasingly popular and general advantages have been extensively highlighted elsewhere. ${ }^{[76-78]}$ Self-optimizing flow platforms using feedback in flow ${ }^{[31,79-}$ ${ }^{81]}$ and autonomous synthesis, which both rely on advanced computational methods, are improving rapidly. ${ }^{[82-85]}$ Platforms which merge both flow chemistry and autonomous synthesis were developed recently, ${ }^{[86]}$ however, high quality (flow) synthesis data is still a major bottleneck in this process.

Flow-based peptide synthesis could serve as a platform to demonstrate the potential of deep learning on automatically collected high-quality synthesis data. ${ }^{[62]}$ In peptide synthesis a single reaction is performed between two out of twenty possible canonical amino acids, leading to 400 possible coupling combinations $^{[68]}$. In addition, peptide synthesis is sequence-dependent, which complicates tailoring reaction conditions even further. With an automatically collected high quality data set new insights on peptide synthesis can be gained and might improve our understanding on sequence-dependent events such as aggregation. ${ }^{[62]}$ The ability to predict 'difficult couplings' with greater confidence would be an important first step toward their correction.

Collection of additional synthesis data might enable the prediction of a highly tailored sequencespecific recipe, which would allow for the synthesis of longer peptides and reduce the need for resource-intensive optimization. To put this into practice it is required to collect more data with AFPS, including additional coupling conditions, backbone modifications and incorporation of new building blocks such as alternative protecting groups and non-canonical amino acids. As discussed earlier, some difficult couplings cannot be suppressed by varying coupling conditions and understanding the impact of sidechain and backbone protecting groups will be crucial to push the limits of peptide synthesis even further. 


\subsection{Mircofluidic Systems}

Over the past two decades, multiple reports on solid-phase and solution-phase syntheses of linear and cyclic peptides using micro-flow (reaction space $<1 \mathrm{~mm}$ ) technology were reported, which have already been extensively reviewed elsewhere ${ }^{[11,87,88]}$ For example, Wang and co-workers reported on a borosilicate glass microchip system for microflow-based SPPS. The chip included an integrated reactor for the solid support (width: $600 \mathrm{~mm}$, length: $1.3 \mathrm{~cm}$ ), demonstrating the possibility of scaling down previously described AFPS systems. ${ }^{[89]}$

In microfluidic systems heat and mass transfer are increased further, which generally leads to more efficient reactions and shorter heating times. In addition, a small-scale system would enable the rapid screening of reaction conditions, which would generate more data to improve our understanding of sequence-specific events while minimizing reagent use. However, per definition microfluidic reactors have a small inner diameter. How much can we scale down AFPS technology? Will AFPS be more efficient in a microfluidic system? And will the decreased diameter lead to issues with backpressure?

\subsection{Green Chemistry}

As environmental factors are becoming increasingly important, there is a growing interest to develop more sustainable alternatives to traditional SPPS reagents, solvents and peptide purification. ${ }^{\text {[90-93] }}$ The E-factor for amide-bond formation is very low and improvements are urgently needed, especially considering that this reaction is amongst the most commonly utilized transformations in the pharmaceutical industry. ${ }^{[94-96]}$ Current strategies rely on the use of hazardous solvents (DMF and Nmethyl-2-pyrrolidone, NMP) and stoichiometric activating or coupling reagents resulting in poor atom economy and accumulation of toxic waste. ${ }^{[97,98]}$ Atom economy is especially low for AFPS, which employs up to 100 equivalents of amino acid for each coupling cycle. In addition, there is accumulating evidence for the health risk of uronium-based coupling agents, ${ }^{[99]}$ which provides additional motivation for new amide bond-forming strategies.

Fülöp and co-workers optimized their flow-based peptide synthesizer with the aim to reduce coupling reagents (HATU), solvents (DMF) and waste. ${ }^{[100]}$ Their set up used a heated reactor $\left(70{ }^{\circ} \mathrm{C}\right)$, which was additionally pressurized (60 bar). Amino acid equivalents needed to achieve high coupling yields were decreased to 1.5-3.0 equivalents in a mesoscale synthesizer and the synthesis of multiple short peptides was demonstrated. ${ }^{[100,101]}$ How much could coupling equivalents and solvents be reduced in AFPS? Can "greener" and potentially less toxic coupling agents such as (1-cyano-2-ethoxy-2oxoethylidenaminooxy)dimethylamino-morpholino-carbenium hexafluorophosphate (COMU), fluoro- $N, N, N^{\prime}, N^{\prime}$-tetramethylformamidinium hexafluorophosphate (TFFH) derivatives or 1propanephosphonic anhydride (T3P) be used in AFPS , too ${ }^{[92]}$ Can solvent used for resin washing be reduced further, as previously described for microwave-based SPPS ${ }^{[102]}$

In addition to minimized reagent usage, greener solvents are also investigated to enable more sustainable methods for peptide synthesis. ${ }^{[93,103]}$ The traditional solvents for amide bond formation are DMF, dimethylacetamide (DMAc) and NMP, all of which are highly problematic in terms of health 
and environmental impact. ${ }^{[104]}$ Replacement of these aprotic, polar solvents proved to be challenging and was the topic of numerous investigations in green chemistry programs, ultimately resulting in numerous recommendations including 2-methyltetrahydrofuran (2-Me-THF), ${ }^{[103]}$ propylene carbonate (PC) ${ }^{[105]}$ dimethyl carbonate (DMC) ${ }^{[103]} \gamma$-valerolactone $(\mathrm{GVL}),{ }^{[106]}$ or $N$-butylpyrrolidinone (NBP) ${ }^{[107]}$ as possible alternatives. As automated fast-flow peptide synthesis allows for rapid and highly reproducible synthesis as well as in-line analysis, AFPS could be an ideal method for a comparative study and analysis of these solvents for SPPS.

The biggest impact could come from a more substantial change to the current process of SPPS — by adapting the entire method to the "ideal" solvent water, rather than adapting the solvent to the current protecting group (Fmoc/t-Bu) chemistry. ${ }^{[93]}$ The first efforts on water-based SPPS were reported by Kawasaki and co-workers, who developed water-compatible coupling agents and protecting groups. ${ }^{[108-115]}$ Notably, PEG-based CM-resin, which is often used in flow-based SPPS, and several related resins show good swelling properties in water. To further improve the properties of watercompatible protecting groups, $\mathrm{SO}_{3}$-functionalized protecting groups, so-called 9-(2sulfo)fluorenylmethyloxycarbonyl (Smoc) amino acid derivatives, ${ }^{[16]}$ as well as additional solvation strategies, additional compatible activators and microwave irradiation were successfully implemented..$^{108,117-123]}$

\subsection{Expanding the reaction tool box on solid support}

Much research is focused on the development of alternative reactions for amide bond formation ${ }^{[98]}$ and flow chemistry could give access to reactions which are difficult or not possible using traditional microwave or batch methods. The rapid consumption of in-line generated highly reactive intermediates through flash chemistry ${ }^{[23,124,125]}$ allows for alternative amino acid activation, e.g. by using more atomeconomical activation or catalytic amide bond formation methods. So far the use of $N$ carboxyanhydrides (NCAs), ${ }^{[126]}$ unsymmetrical acid anhydrides, acyl azides ${ }^{[127]}$ and symmetric anhydrides ${ }^{[128]}$ has been demonstrated using flow chemistry, however, most of these were reported in the context of liquid phase peptide synthesis. Very recently Fuse and co-workers reported on the use of highly active acyl $\mathrm{N}$-methylimidazolium cations in for amide bond formation in micro-flow, which was also applicable to SPPS. ${ }^{[129]}$

Can these methods be used in AFPS and can we develop even more reactive active esters? Can catalytical methods for amide bond formation ${ }^{[130-134,135]}$ be translated into flow chemistry? And can these methods be used for the synthesis of long peptides or even proteins?

Flow-based stepwise synthesis on solid support was developed and optimized for several biopolymers, including polysaccharides, ${ }^{[136,137]} \mathrm{DNA} / \mathrm{RNA}$ and peptides/proteins. ${ }^{[14]}$ Precise reaction parameter control and decreased reaction times allow for a rapid screening of conditions and often result in an increased yield per incorporated monomer. Lessons learned in the development of the afore mentioned methods could be translated to other (artificial) sequence defined polymers (SDP), such as peptoids, antisense-oligonucleotides, oligourea foldamers ${ }^{[138]}$ or aromatic oligoamide foldamers. ${ }^{[139,140]}$ We therefore anticipate that the developments of new reactions for solid phase flow chemistry inside and outside of peptide-related research — will have great impact on SDP research. 


\section{Conclusion}

In just a few years time, the advent of in-line heating and reagent activation has transformed flowbased SPPS from a largely forgotten approach to the 'cutting edge' of solid phase synthesis. Where AFPS instrumentation is available, it will streamline chemical protein synthesis by furnishing the requisite peptide segments for chemical ligation, or in some cases the full-length protein chains directly. In addition, AFPS is generating ever-expanding sets of analytical data that could impact SPPS more broadly. As aspects of this data challenge existing notions (e.g., the onset of chain aggregation in SPPS) or are not readily explained using established concepts (e.g., the frequently-incomplete coupling of Fmoc-Asn(Trt)-OH), future developments will be watched with great interest. The fundamentals of SPPS are established, but new developments should not be precluded, and we look forward to continued progress at the forefront of chemical synthesis.

\section{Acknowledgements}

We would like to thank Steve Kent, Fernando Albericio, Ethan Evans, Alex Mijalis, Brad Pentelute, and our anonymous reviewers for helpful comments on this manuscript, Alex Vinogradov and Somesh Mohapatra for insightful discussions, and the many Pentelute lab alumni who contributed to the 'renaissance' of flow-based peptide synthesis in Cambridge, MA. Zak gratefully acknowledges the support of Charles Johannes, Sir David Lane, and the Peptide Engineering Program at A*STAR (Grant ID H17/01/a0/010).

\section{ORCID}

Nina Hartrampf: 0000-0003-0875-6390

\section{Conflict of Interest}

Zak was a Ph.D. student in the group of Steve Kent, a postdoctoral researcher in the group of Brad Pentelute, and is a co-author on the manuscript "Synthesis of proteins by automated flow chemistry" (Science $2020,368,980-9879)$. Nina was a postdoctoral researcher in the group of Brad Pentelute and is a coauthor on the manuscripts "Synthesis of proteins by automated flow chemistry" (Science 2020, 368, 980-9879) and "Deep Learning for Prediction and Optimization of Fast-Flow Peptide Synthesis" (Preprint on ChemRxiv: https://doi.org/10.26434/chemrxiv.12649343.v1). Both authors declare that all opinions in this perspective article are their own.

\section{References:}

[1] S. B. H. Kent, Protein Sci. 2019, 28, 313-328.

[2] P. Dawson, T. Muir, I. Clark-Lewis, S. Kent, Science 1994, 266, 776.

[3] M. Schnolzer, S. Kent, Science 1992, 256, 221.

[4] P. E. Dawson, S. B. H. Kent, Annu. Rev. Biochem. 2000, 69, 923-960.

[5] S. B. H. Kent, Chem. Soc. Rev. 2009, 38, 338-351.

[6] A. El-Faham, F. Albericio, Chem. Rev. 2011, 111, 6557-6602.

[7] V. Agouridas, O. El Mahdi, V. Diemer, M. Cargoët, J.-C. M. Monbaliu, O. Melnyk, Chem. Rev. 2019, 119, 7328-7443.

[8] E. Fischer, Synthese von Derivaten der Polypeptide. In: Untersuchungen über Aminosäuren, Polypeptide und Proteïne (1899-1906). Springer, Berlin, Heidelberg, 1906.

[9] T. Kimmerlin, D. Seebach, J. Peptide. Res. 2005, 65, 229-260. 
[10] R. Behrendt, P. White, J. Offer, J. Pept. Sci. 2016, 22, 4-27

[11] C. P. Gordon, Org. Biomol. Chem. 2018, 16, 180-196.

[12] A. J. Mijalis, D. A. Thomas III, M. D. Simon, A. Adamo, R. Beaumont, K. F. Jensen, B. L. Pentelute, Nat. Chem. Biol. 2017, 13, 464.

[13] M. D. Simon, P. L. Heider, A. Adamo, A. A. Vinogradov, S. K. Mong, X. Li, T. Berger, R. L. Policarpo, C. Zhang, Y. Zou, et al., ChemBioChem 2014, 15, 713-720.

[14] N. Hartrampf, A. Saebi, M. Poskus, Z. P. Gates, A. J. Callahan, A. E. Cowfer, S. Hanna, S. Antilla, C. K. Schissel, A. J. Quartararo, X. Ye, A. J. Mijalis, M. D. Simon, A. Loas, S. Liu, C. Jessen, T. E. Nielsen, B. L. Pentelute, Science 2020, 368, 980-987.

[15] J. Servais, C. Lambert, E. Fontaine, J.-M. Plesséria, I. Robert, V. Arendt, T. Staub, F. Schneider, R. Hemmer, G. Burtonboy, J.-C. Schmit, J. Clin. Microbiol. 2001, 39, 454-459.

[16] E. C. Johnson, E. Malito, Y. Shen, D. Rich, W. J. Tang, S. B. Kent, J. Am. Chem. Soc. 2007, 129, 11480-11490.

[17] E. Bayer, G. Jung, I. Halász, I. Sebestian, Tetrahedron Lett. 1970, 11, 4503-4505.

[18] R. B. Merrifield, in Prog. Pept. Res. Vol. II Proc. Second Am. Pept. Symp., Gordon And Breach, Science Publishers, Inc., New York, 1970, pp. 89-92.

[19] E. Bayer, Angew. Chem. Int. Ed. Engl. 1991, 30, 113-129.

[20] E. Atherton, E. Brown, R. C. Sheppard, A. Rosevear, J Chem Soc Chem Commun 1981, 11511152.

[21] T. J. Lukas, M. B. Prystowsky, B. W. Erickson, Proc. Natl. Acad. Sci. 1981, 78, 2791.

[22] R. P. W. Scott, K. K. Chan, P. Kucera, S. Zolty, J. Chromatogr. Sci. 1971, 9, 577-591.

[23] J. Yoshida, Y. Takahashi, A. Nagaki, Chem. Commun. 2013, 49, 9896-9904.

[24] A. Dryland, R. C. Sheppard, J. Chem. Soc. Perkin 1 1986, 125-137.

[25] H. Lahm, S. Udenfriend, J. Meienhofer, in Pept. Struct. Funct. Proc. Eighth Am. Pept. Symp., Pierce Chemical Company, Rockford, Illinois, US, 1983, pp. 69-72.

[26] V. Krchnak, J. Vagner, J. Eichler, M. Lebl, Peptides 1988, 232-234.

[27] R. Frank, H. Leban, M. Kraft, H. Gausepohl, in Pept. Chem. Biol. Proc. Tenth Am. Pept. Symp., ESCOM, Leiden, The Netherlands, 1988, pp. 215-216.

[28] W. Rapp, H. Fritz, E. Bayer, in Pept. Chem. Biol. Proc. Twelfth Am. Pept. Symp., ESCOM, Leiden, The Netherlands, 1992, pp. 529-530.

[29] L. R. Cameron, J. L. Holder, M. Meldal, R. C. Sheppard, J. Chem. Soc. Perkin 1 1988, 2895-2901.

[30] E. Atherton, A. Dryland, R. C. Sheppard, J. D. Wade, in Pept. Struct. Funct. Proc. Eighth Am. Pept. Symp., Pierce Chemical Company, Rockford, Illinois, US, 1983.

[31] B. J. Reizman, K. F. Jensen, Acc. Chem. Res. 2016, 49, 1786-1796.

[32] J. Bedford, C. Hyde, T. Johnson, W. Jun, D. Owen, M. Quibell, R. Sheppard, Int. J. Pept. Protein Res. 1992, 40, 300-307.

[33] V. K. Sarin, S. B. H. Kent, R. B. Merrifield, J. Am. Chem. Soc. 1980, 102, 5463-5470.

[34] I. L. Rodionov, M. Baru, V. Ivanov, Pept. Res 1992, 5, 119-125.

[35] M. Baru, L. Mustaeva, I. Vagenina, E. Y. Gorbunova, V. Cherskii, J. Pept. Res. 2001, 57, 193202.

[36] I. L. Rodionov, I. A. Peshenko, L. K. Baidakova, V. T. Ivanov, Int. J. Pept. Res. Ther. 2007, 13, 161-171.

[37] E. T. Sletten, M. Nuño, D. Guthrie, P. H. Seeberger, Chem. Commun. 2019, DOI 10.1039/C9CC08421E.

[38] F. Albericio, S. Alefelder, M. Amblard, V. Antonenko, E. Atherton, G. Barany, G. Becker, N. L. Benoiton, D. Besser, M. Beyermann, et al., Houben-Weyl Methods of Organic Chemistry Vol. E 22a, 4th Edition Supplement, Georg Thieme Verlag, Stuttgart, New York, 2004.

[39] A. Pernille Tofteng, S. L. Pedersen, D. Staerk, K. J. Jensen, Chem. - Eur. J. 2012, 18, 9024 9031. 
[40] L. K. Spare, V. Laude, D. G. Harman, J. R. Aldrich-Wright, C. P. Gordon, React. Chem. Eng. 2018, 3, 875-882.

[41] T. Handel, W. F. DeGrado, J. Am. Chem. Soc. 1990, 112, 6710-6711.

[42] C.-R. Wu, V. C. Stevens, G. W. Tregear, J. D. Wade, J. Chem. Soc. Perkin 1 1989, 81-87.

[43] C. Valembois, C. Mendre, J. C. Cavadore, B. Calas, Tetrahedron Lett. 1992, 33, 4005-4008.

[44] E. Bianchi, M. Sollazzo, A. Tramontano, A. Pessi, Int. J. Pept. Protein Res. 1993, 41, 385-393.

[45] I. Clark-Lewis, R. Aebersold, H. Ziltener, J. W. Schrader, L. E. Hood, S. B. Kent, Science 1986, 231, 134-139.

[46] P. White, J. W. Keyte, K. Bailey, G. Bloomberg, J. Peptide Sci. 2004, 10, 18-26.

[47] S. Frutos, J. Tulla-Puche, F. Albericio, E. Giralt, Int. J. Pept. Res. Ther. 2007, 13, 221-227.

[48] I. Clark-Lewis, L. E. Hood, S. B. Kent, Proc. Natl. Acad. Sci. 1988, 85, 7897-7901.

[49] A. Wlodawer, M. Miller, M. Jaskolski, B. K. Sathyanarayana, E. Baldwin, I. T. Weber, L. M. Selk, L. Clawson, J. Schneider, S. B. Kent, Science 1989, 245, 616-621.

[50] M. Schnölzer, P. Alewood, A. Jones, D. Alewood, S. B. Kent, Int. J. Pept. Protein Res. 1992, 40, 180-193.

[51] S. B. Kent, P. F. Alewood, Synth. Biol. - Synth. Biomol. 2014, 22, viii-xi.

[52] S. K. Mong, A. A. Vinogradov, M. D. Simon, B. L. Pentelute, ChemBioChem 2014, 15, 721-733.

[53] W. Lu, M. A. Qasim, S. B. H. Kent, J. Am. Chem. Soc. 1996, 118, 8518-8523.

[54] A. R. Mitchell, Pept. Sci. 2008, 90, 215-233.

[55] S. B. H. Kent, D. Alewood, P. Alewood, M. Baca, A. Jones, M. Schnölzer, in Innov. Perspect. Solid Phase Synth. Pept. Polypept. Oligonucleotides, Intercept Limited, Andover, 1992, pp. 407-414.

[56] S. B. Kent, Annu. Rev. Biochem. 1988, 57, 957-989.

[57] S. B. H. Kent, in Pept. Struct. Funct. Proc. Ninth Am. Pept. Symp., Pierce Chemical Company, Rockford, Illinois, US, 1985, pp. 407-414.

[58] J. Schneider, S. B. H. Kent, Cell 1988, 54, 363-368.

[59] S. M. Meister, S. B. H. Kent, in Pept. Struct. Funct. Proc. Eighth Am. Pept. Symp., Pierce Chemical Company, Rockford, Illinois, US, 1983, pp. 103-106.

[60] E. Atherton, R. C. Sheppard, in Pept. Struct. Funct. Proc. Ninth Am. Pept. Symp., Pierce Chemical Company, Rockford, Illinois, US, 1985, pp. 415-418.

[61] M. Paradís-Bas, J. Tulla-Puche, F. Albericio, Chem. Soc. Rev. 2016, 45, 631-654.

[62] S. Mohapatra, N. Hartrampf, M. Poskus, A. Loas, R. Gómez-Bombarelli, B. L. Pentelute, 2020, Preprint on ChemRxiv: https://doi.org/10.26434/chemrxiv.12649343.v1

[63] D. Lloyd, G. Petrie, R. Noble, J. Tam, Pept. Chem. Struct. Biol. Proc. 11th Am. Pept. Symp. Rivier JE Marshall GR Eds Escom Leiden Neth. 1990, 909-910.

[64] K. Barlos, D. Papaioannou, S. Patrianakou, T. Tsegenidis, Liebigs Ann. Chem. 1986, 1986, $1950-$ 1955.

[65] J. Tam, in Pept. Struct. Funct. Proc. Ninth Am. Pept. Symp., Pierce Chemical Company, Rockford, Illinois, US, 1985, pp. 423-425.

[66] A. J. Mijalis, A. Steinauer, A. Schepartz, B. L. Pentelute, in Flow Chem. Org. Synth. (Eds.: T.F. Jamison, G. Koch), Georg Thieme Verlag, Stuttgart, 2018, pp. 381-398.

[67] A.-B. M. Abdel-Aal, G. Papageorgiou, M. Quibell, J. Offer, Chem. Commun., 2014, 50, 8316-8319.

[68] DS Kemp, SH Choong, and J Pekaar, J. Org. Chem., 1974, 39(26):3841-3847.

[69] W. J. Van Woerkom, J. W. Van Nispen, Int. J. Pept. Protein Res. 1991, 38, 103-113.

[70] M. Cezari, L. Juliano, Pept. Res. 1996, 9, 88-91.

[71] E. Valeur, M. Bradley, Chem. Soc. Rev. 2009, 38, 606-631.

[72] J. Hachmann, M. Lebl, Pept. Sci. Orig. Res. Biomol. 2006, 84, 340-347.

[73] M. Mergler, F. Dick, B. Sax, P. Weiler, T. Vorherr, J. Pept. Sci. 2003, 9, 36-46.

[74] K. Neumann, J. Farnung, S. Baldauf, J. Bode, Nat. Comm. 2020, 11, 982. 
[75] T. Michels, R. Dölling, U. Haberkorn, W. Mier, Org. Lett. 2012, 14, 5218-5221.

[76] J. Britton, C. L. Raston, Chem. Soc. Rev. 2017, 46, 1250-1271.

[77] M. B. Plutschack, B. Pieber, K. Gilmore, P. H. Seeberger, Chem. Rev. 2017, 117, 11796-11893.

[78] D. Webb, T. F. Jamison, Chem. Sci. 2010, 1, 675-680.

[79] A.-C. Bédard, A. Adamo, K. C. Aroh, M. G. Russell, A. A. Bedermann, J. Torosian, B. Yue, K. F. Jensen, T. F. Jamison, Science 2018, 361, 1220.

[80] S. Steiner, J. Wolf, S. Glatzel, A. Andreou, J. M. Granda, G. Keenan, T. Hinkley, G. AragonCamarasa, P. J. Kitson, D. Angelone, et al., Science 2019, 363, eaav2211.

[81] D. E. Fitzpatrick, C. Battilocchio, S. V. Ley, Org. Process Res. Dev. 2016, 20, 386-394.

[82] K. F. Jensen, C. W. Coley, N. S. Eyke, Angew. Chem. Int. Ed. 2019, 0, DOI 10.1002/anie.201909987.

[83] C. W. Coley, N. S. Eyke, K. F. Jensen, Angew. Chem. Int. Ed. 2019, 0, DOI 10.1002/anie.201909989.

[84] C. W. Coley, W. H. Green, K. F. Jensen, Acc. Chem. Res. 2018, 51, 1281-1289.

[85] S. Szymkuć, E. P. Gajewska, T. Klucznik, K. Molga, P. Dittwald, M. Startek, M. Bajczyk, B. A. Grzybowski, Angew. Chem. Int. Ed. 2016, 55, 5904-5937.

[86] C. W. Coley, D. A. Thomas, J. A. M. Lummiss, J. N. Jaworski, C. P. Breen, V. Schultz, T. Hart, J. S. Fishman, L. Rogers, H. Gao, et al., Science 2019, 365, eaax1566.

[87] S. Fuse, Y. Otake, H. Nakamura, Chem. Asian J. 2018, 13, 3818-3832.

[88] S. Ramesh, P. Cherkupally, B. G. de la Torre, T. Govender, H. G. Kruger, F. Albericio, Amino Acids 2014, 46, 2091-2104.

[89] W. Wang, Y. Huang, J. Liu, Y. Xie, R. Zhao, S. Xiong, G. Liu, Y. Chen, H. Ma, Lab. Chip 2011, 11, 929-935.

[90] S. Datta, A. Sood, M. Torok, Curr. Org. Synth. 2011, 8, 262-280.

[91] Y. E. Jad, A. Kumar, A. El-Faham, B. G. de la Torre, F. Albericio, ACS Sustain. Chem. Eng. 2019, 7, 3671-3683.

[92] A. Isidro-Llobet, M. N. Kenworthy, S. Mukherjee, M. E. Kopach, K. Wegner, F. Gallou, A. G. Smith, F. Roschangar, J. Org. Chem. 2019, 84, 4615-4628.

[93] O. Al Musaimi, B. G. de la Torre, F. Albericio, Green Chem. 2020, 22, 996-1018.

[94] M. C. Bryan, P. J. Dunn, D. Entwistle, F. Gallou, S. G. Koenig, J. D. Hayler, M. R. Hickey, S. Hughes, M. E. Kopach, G. Moine, et al., Green Chem. 2018, 20, 5082-5103.

[95] D. J. C. Constable, P. J. Dunn, J. D. Hayler, G. R. Humphrey, Jr. Leazer Johnnie L., R. J. Linderman, K. Lorenz, J. Manley, B. A. Pearlman, A. Wells, et al., Green Chem. 2007, 9, 411420.

[96] K. G. Varnava, V. Sarojini, Chem. - Asian J. 2019, 0, DOI 10.1002/asia.201801807.

[97] K. E. Schwieter, J. N. Johnston, J. Am. Chem. Soc. 2016, 138, 14160-14169.

[98] V. R. Pattabiraman, J. W. Bode, Nature 2011, 480, 471.

[99] K. J. McKnelly, W. Sokol, J. S. Nowick, J. Org. Chem. 2020, 85, 1764-1768.

[100] I. M. Mándity, B. Olasz, S. B. Ötvös, F. Fülöp, ChemSusChem 2014, 7, 3172-3176.

[101] A. Szloszár, F. Fülöp, I. M. Mándity, Chemistry Select 2017, 2, 6036-6039.

[102] J. M. Collins, K. A. Porter, S. K. Singh, G. S. Vanier, Org. Lett. 2014, 16, 940-943.

[103] D. S. MacMillan, J. Murray, H. F. Sneddon, C. Jamieson, A. J. B. Watson, Green Chem. 2013, 15, 596-600.

[104] F. P. Byrne, S. Jin, G. Paggiola, T. H. M. Petchey, J. H. Clark, T. J. Farmer, A. J. Hunt, C. Robert McElroy, J. Sherwood, Sustain. Chem. Process. 2016, 4, 7.

[105] S. B. Lawrenson, R. Arav, M. North, Green Chem. 2017, 19, 1685-1691.

[106] A. Kumar, Y. E. Jad, J. M. Collins, F. Albericio, B. G. de la Torre, ACS Sustain. Chem. Eng. 2018, 6, 8034-8039. 
[107] J. Lopez, S. Pletscher, A. Aemissegger, C. Bucher, F. Gallou, Org. Process Res. Dev. 2018, 22, 494-503.

[108] K. Hojo, H. Ichikawa, A. Hara, M. Onishi, K. Kawasaki, Y. Fukumori, Protein Pept. Lett. 2012, 19, 1231-1236.

[109] K. Hojo, M. Maeda, N. Tanakamaru, K. Mochida, K. Kawasaki, Protein Pept Lett 2006, 13, 189.

[110] K. Hojo, M. Maeda, K. Kawasaki, J. Pept. Sci. 2001, 7, 615-618.

[111] K. Hojo, M. Maeda, Y. Takahara, S. Yamamoto, K. Kawasaki, Tetrabedron Lett. 2003, 44, 2849_ 2851.

[112] K. Hojo, M. Maeda, K. Kawasaki, Tetrahedron 2004, 60, 1875-1886.

[113] K. Hojo, M. Maeda, T. J. Smith, E. Kita, F. Yamaguchi, S. Yamamoto, K. Kawasaki, Chem. Pharm. Bull. (Tokyo) 2004, 52, 422-427.

[114] K. Hojo, M. Maeda, K. Kawasaki, Tetrahedron Lett. 2004, 45, 9293-9295.

[115] K. Kawasaki, T. Tsuji, M. Maeda, T. Matsumoto, K. Hirase, Chem. Pharm. Bull. (Tokyo) 1987, 35, $1044-1048$.

[116] R. B. Merrifield, A. E. Bach, J. Org. Chem. 1978, 43, 4808-4816.

[117] S. Knauer, T. M. L. Roese, O. Avrutina, H. Kolmar, C. Uth, Method for Peptide Synthesis and Apparatus for Carrying out a Method for Solid Phase Synthesis of Peptides, n.d., EP3201216A1.

[118] A. S. Galanis, F. Albericio, M. Grøtli, Org. Lett. 2009, 11, 4488-4491.

[119] K. Hojo, H. Ichikawa, M. Maeda, S. Kida, Y. Fukumori, K. Kawasaki, J. Pept. Sci. Off. Publ. Eur. Pept. Soc. 2007, 13, 493-497.

[120] C. M. Gabriel, M. Keener, F. Gallou, B. H. Lipshutz, Org. Lett. 2015, 17, 3968-3971.

[121] Q. Wang, Y. Wang, M. Kurosu, Org. Lett. 2012, 14, 3372-3375.

[122] F. Gallou, P. Guo, M. Parmentier, J. Zhou, Org. Process Res. Dev. 2016, 20, 1388-1391.

[123] S. Knauer, C. Uth, N. Koch, R. Meusinger, O. Avrutina, H. Kolmar, Angew. Chem. Int. Ed. 2020, 59, 12984-12990.

[124] J. Yoshida, Chem. Rec. 2010, 10, 332-341.

[125] J. Yoshida, A. Nagaki, T. Yamada, Chem. Eur. J. 2008, 14, 7450-7459.

[126] R. De Marco, A. Tolomelli, A. Greco, L. Gentilucci, ACS Sustain. Chem. Eng. 2013, 1, 566-569.

[127] A. Mata, U. Weigl, O. Flögel, P. Baur, C. A. Hone, C. O. Kappe, React. Chem. Eng. 2020, 5, 645-650.

[128] S. Fuse, Y. Mifune, T. Takahashi, Angew. Chem. Int. Ed. 2014, 53, 851-855.

[129] Y. Otake, Y. Shibata, Y. Hayashi, S. Kawauchi, H. Nakamura, S. Fuse, Angew. Chem. Int. Ed. early view, 10.1002/anie.202002106.

[130] Handoko, S. Satishkumar, N. R. Panigrahi, P. S. Arora, J. Am. Chem. Soc. 2019, 141, 1597715985.

[131] W. Muramatsu, H. Tsuji, H. Yamamoto, ACS Catal. 2018, 8, 2181-2187.

[132] P. Huy, B. Zoller, Nachrichten Aus Chem. 2019, DOI 10.1002/nadc.20194087418).

[133] Y. Du, T. Barber, S. E. Lim, H. S. Rzepa, I. R. Baxendale, A. Whiting, Chem Commun 2019, 55, 2916-2919.

[134] M. T. Sabatini, L. T. Boulton, T. D. Sheppard, Sci. Adv. 2017, 3, e1701028.

[135] Z. Liu, H. Noda, M. Shibasaki, N. Kumagai, Org. Lett. 2018, 20, 612-615.

[136] A. A. Joseph, A. Pardo-Vargas, P. H. Seeberger, J. Am. Chem. Soc. 2020, DOI 10.1021/jacs.0c00751.

[137] H. S. Hahm, M. Hurevich, P. H. Seeberger, Nat. Commun. 2016, 7, 12482.

[138] P. Nanjan, M. Porel, Polym. Chem. 2019, 10, 5406-5424.

[139] B. Baptiste, C. Douat-Casassus, K. Laxmi-Reddy, F. Godde, I. Huc, J. Org. Chem. 2010, 75, 7175-7185.

[140] E. R. Gillies, F. Deiss, C. Staedel, J.-M. Schmitter, I. Huc, Angew. Chem. Int. Ed. 2007, 46, 4081-4084. 